\title{
The weak Fe fluorescence line and long-term X-ray evolution of the Compton-thick AGN in NGC 7674
}

\author{
P. Gandhi, ${ }^{1,2}$ A. Annuar, ${ }^{2}$ G.B. Lansbury, ${ }^{2,3}$ D. Stern, ${ }^{4}$ D.M. Alexander, ${ }^{2}$ F.E. Bauer, ${ }^{5,6,7}$ \\ S. Bianchi, ${ }^{8}$ S.E. Boggs,${ }^{9}$ P.G. Boorman, ${ }^{1}$ W.N. Brandt, ${ }^{10,11,12}$ M. Brightman, ${ }^{13}$ \\ F.E. Christensen, ${ }^{14}$ A. Comastri,${ }^{15}$ W.W. Craig, ${ }^{14,16}$ A. Del Moro, ${ }^{17}$ M. Elvis, ${ }^{18}$ \\ M. Guainazzi, ${ }^{19,20}$ C.J. Hailey, ${ }^{21}$ F.A. Harrison, ${ }^{13}$ M. Koss, ${ }^{22}$ I. Lamperti, ${ }^{22}$ G. Malaguti, ${ }^{23}$ \\ A. Masini, ${ }^{15,24}$ G. Matt, ${ }^{8}$ S. Puccetti,${ }^{25,26}$ C. Ricci, ${ }^{5}$ E. Rivers, ${ }^{13}$ D.J. Walton ${ }^{3,4,13}$, \\ W.W. Zhang ${ }^{27}$ \\ Author affiliations appear at the end of the paper.
}

18 October 2018

\begin{abstract}
We present NuSTAR X-ray observations of the active galactic nucleus (AGN) in NGC 7674. The source shows a flat X-ray spectrum, suggesting that it is obscured by Compton-thick gas columns. Based upon long-term flux dimming, previous work suggested the alternate possibility that the source is a recently switched-off AGN with the observed X-rays being the lagged echo from the torus. Our high-quality data show the source to be reflection-dominated in hard $\mathrm{X}$-rays, but with a relatively weak neutral $\mathrm{Fe} \mathrm{K} \alpha$ emission line (equivalent width [EW] of $\approx 0.4 \mathrm{keV})$ and a strong Fe XXVI ionised line $(\mathrm{EW} \approx 0.2 \mathrm{keV})$. We construct an updated longterm X-ray light curve of NGC 7674 and find that the observed 2-10 keV flux has remained constant for the past $\approx 20$ years, following a high flux state probed by Ginga. Light travel time arguments constrain the minimum radius of the reflector to be $\sim 3.2 \mathrm{pc}$ under the switchedoff AGN scenario, $\approx 30$ times larger than the expected dust sublimation radius, rendering this possibility unlikely. A patchy Compton-thick AGN (CTAGN) solution is plausible, requiring a minimum line-of-sight column density $\left(N_{\mathrm{H}}\right)$ of $3 \times 10^{24} \mathrm{~cm}^{-2}$ at present, and yields an intrinsic 2-10 keV luminosity of (3-5) $\times 10^{43} \mathrm{erg} \mathrm{s}^{-1}$. Realistic uncertainties span the range of $\approx(1-13) \times 10^{43} \mathrm{erg} \mathrm{s}^{-1}$. The source has one of the weakest fluorescence lines amongst bona fide CTAGN, and is potentially a local analogue of bolometrically luminous systems showing complex neutral and ionised $\mathrm{Fe}$ emission. It exemplifies the difficulty of identification and proper characterisation of distant CTAGN based on the strength of the neutral $\mathrm{Fe} \mathrm{K} \alpha$ line.
\end{abstract}

Key words: Seyfert - X-rays: individual (NGC 7674)

\section{INTRODUCTION}

Obscured active galactic nuclei (AGN) dominate the overall population of AGN in the cosmos, especially the efficiently-accreting sources which power the cosmic hard X-ray background radiation (e.g. Setti \& Woltier 1989; Comastri et al. 1995; Gandhi \& Fabian 2003; Gilli et al. 2007; Treister et al. 2009; Ballantyne et al. 2011; Ueda et al. 2014). Yet, finding and characterising these objects is made difficult by the strong absorption they can suffer across the electromagnetic spectrum. In particular, the census of the sources hidden behind extreme obscuring column densities of gas - in particular Compton-thick AGN with column densities $N_{\mathrm{H}} \gtrsim 1.5 \times 10^{24} \mathrm{~cm}^{-2}$; hereafter, CTAGN - remains highly incomplete (see Ricci et al. 2015 and Koss et al. 2016 for recent updates on the hard X-ray selected CTAGN census). Even locally, very few robust (bona fide) CTAGN are known (Della Ceca et al. 2008; Goulding et al. 2012; Gandhi et al. 2014).

One candidate of a nearby, luminous CTAGN is NGC 7674, the brightest member of the Hickson 96 interacting galaxy group. NGC 7674 is a known Seyfert 2, showing strong narrow optical emission lines with full widths at half maximum (FWHM) of less than $500 \mathrm{~km} \mathrm{~s}^{-1}$ in spectral observations carried out over three decades ago (Feldman et al. 1982). The source also shows a prolific outflow, which manifests as a prominent shoulder on the blue wings of all the prominent narrow optical lines. The outflow has been studied in detail using HST spectroscopy by Fischer et al. (2013), who find blueshifts of up to $\sim 1700 \mathrm{~km} \mathrm{~s}^{-1}$ (and even larger FWHM) along the narrow line region aligned with the jet axis of the source. In the radio, NGC 7674 shows at least three separate compact components in VLBI observations on scales of $\approx 0.7 \operatorname{arcsec}$ 
corresponding to $\approx 0.4 \mathrm{kpc}$, and there have been suggestions that the collimated ejecta associated with the radio source could also drive the optical-emitting line outflow (Unger et al.|1988). Even higher resolution VLBI observations by Momjian et al. (2003) reveal a complex ' $\mathrm{S}$ '-shaped structure, which could result from interactions of the jet with the interstellar medium. Using the [O III] emission line as a bolometric luminosity indicator, Xu et al. (1999) classify NGC 7674 as a radio quiet AGN.

The source was first reported to be a refection-dominated AGN by Malaguti et al. (1998) from BeppoSAX X-ray observations carried out in 1996, with the direct (intrinsic) continuum being fully absorbed by a Compton-thick gas column. BeppoSAX detected NGC 7674 over the energy range of $\sim 0.1-60 \mathrm{keV}$. Malaguti et al. reported a complex structure to the neutral $\mathrm{Fe} \mathrm{K} \alpha$ line, and estimated a high intrinsic AGN luminosity assuming a scattering geometry similar to NGC 1068. But the BeppoSAX observation was not the first X-ray observation of the source. As detailed in a historical X-ray analysis by Bianchi et al. (2005), the source was likely detected by the HEAO mission in the late 1970s at a 2$10 \mathrm{keV}$ flux level almost 30 times brighter (Grossan 1992) and subsequently with an intermediate flux by Ginga (Awaki et al. 1991). Bianchi et al. (2005) discuss several potential caveats to these detections, including $H E A O$ contamination by nearby sources, and systematic uncertainties related to the background level measured by Ginga, and conclude that the combined weight of evidence favours the historical source detections being real, although the Ginga flux measurement is considered to be more reliable of the two, due to a more robust background determination. The Ginga spectrum is a lightly absorbed power law (with $N_{\mathrm{H}}<2 \times 10^{22} \mathrm{~cm}^{-2}$ ) with an upper limit of $80 \mathrm{eV}$ to the equivalent width (EW) of any neutral $\mathrm{Fe} \mathrm{K} \alpha$ emission line. The source then declined by an order of magnitude in continuum flux by the time it was observed by BeppoSAX to be Compton-thick. Such behaviour might argue for the source being a member of the class of 'changing-look' AGN (e.g., Matt et al. 2003), associated with clumps of obscuring clouds transiting across the line-of-sight (1.o.s) resulting in apparent changes in $N_{\mathrm{H}}$ (1.o.s). The most famous example of this class is NGC 1365 which shows dramatic $N_{\mathrm{H}}$ variability on timescales of days (Risaliti et al. 2005).

However, an XMM-Newton observation carried out 6 years later (in 2004) also showed a reflection-dominated spectrum completely consistent in shape as well as flux with BeppoSAX, unlike what may be expected in a changing-look AGN. Bianchi et al. (2005) interpret the source as potentially having switched-off, with the spectrum observed by BeppoSAX and XMM-Newton being the reflection component which is delayed with respect to the direct, illuminating power law (PL). Another source that has been discussed from these two opposing perspectives recently is NGC 7582 (Rivers et al. 2015). Bianchi et al. (2005) also found a relatively weak Fe K $\alpha$ emission line in NGC 7674, possibly blended with an ionised Fe XXVI line at $6.97 \mathrm{keV}$.

Approximately 10 years after the last XMM-Newton observation, NGC 7674 was observed by Suzaku in 2013 and then by NUSTAR in 2014, in addition to several snapshot observations by the Swift satellite between 2011 to 2014 . Here, we present a spectral analysis of these unpublished observations, and combine this with historical data to study the long-term source evolution. We discuss and place constraints on the switched-off AGN as well as the CTAGN scenarios. Finally, we touch upon the relevance of the complex Fe lines for the identification and characterisation of distant CTAGN. We assume $H_{0}=67.3 \mathrm{~km} \mathrm{~s}^{-1} \mathrm{Mpc}^{-1}$ and $\Omega_{\Lambda}=0.685$ (Planck Collaboration 2014), corresponding to a dis-
Table 1. Observation Log

\begin{tabular}{lccr}
\hline \hline Mission & Instrument(s) & Observation date & $\begin{array}{r}\text { Exposure } \\
\text { ks }\end{array}$ \\
\hline NuSTAR & FPMA/B & $2014-09-30$ & $52.0 / 51.9$ \\
Suzaku & XIS0/1/3 & $2013-12-08$ & $52.2 / 52.2 / 52.2$ \\
Swift & XRT & $2011-01-28 \ldots 2014-10-08^{\dagger}$ & $48.8^{\dagger}$ \\
\hline
\end{tabular}

${ }^{\dagger}$ For Swift, 17 observations are combined here. See Appendix for details.

tance of $126 \mathrm{Mpc}$ corrected to the reference frame defined by the cosmic microwave background. The source systemic redshift is $z=0.0289$. All X-ray spectral fitting is carried out with the XSPEC package v12.9.0 (Arnaud 1996) and uncertainties are quoted at $90 \%$ confidence, unless stated otherwise.

\section{OBSERVATIONS}

A log of the NuSTAR, Suzaku and Swift X-ray observations analysed herein is presented in Table 1 and the individual data sets are described in this section.

\subsection{NUSTAR}

NGC 7674 was observed by NuSTAR (Harrison et al. 2013) for about $52 \mathrm{ks}$ of exposure on 2014 Sep 30 (ObsID 708023010). NuS$T A R$ is the first orbiting telescope capable of focusing $\mathrm{X}$-rays above $\sim 10 \mathrm{keV}$, operating over the energy range of $3-79 \mathrm{keV}$. The data were processed using standard steps and the NuSTAR Data Analysis Software (NUSTARDAS) v.1.3.0 which is provided as part of HEASOFT ${ }^{27}$ and associated FTOOLS (Blackburn 1995). NuSTAR CALDB calibration files were used to generate cleaned event files after filtering for South Atlantic Anomaly passages and the standard depth cut, in order to reduce instrumental background.

Source spectra were extracted using a circular aperture $45^{\prime \prime}$ in radius centred on the source position in both focal plane modules (FPMs). Background spectra were extracted from source free regions on the detector. The nuproduct $s$ task was used to extract these calibrated spectra and to generate corresponding response files. All spectra were grouped to a minimum signal-to-noise of at least 4 per grouped energy bin after background subtraction for fitting purposes.

The source is well detected in both FPMs with $3-78 \mathrm{keV}$ count rates per second of $2.92 \pm 0.08$ (FPMA) and $2.53 \pm 0.08$ (FPMB).

\subsection{Suzaku}

About $52 \mathrm{ks}$ of exposure were obtained on 2013 Dec 08 with Suzaku. The X-ray Imaging Spectrometer (XIS; Koyama et al. 2007) is sensitive over $\approx 0.5-10 \mathrm{keV}$. Standard FTOOLS software for Suzaku was used for data reduction and cleaned event file generation with recommended filtering. Source counts were extracted from within a 3.4 arcmin radius aperture for integrating XIS source counts, and background counts from a larger source-free polygon. The generated spectra and responses of the two front-illuminated

27 https://heasarc.gsfc.nasa.gov 
(FI) detectors were combined together, and this was analysed simultaneously with the back-illuminated (BI) detector data. For the fitting, we ignore the energy ranges of $1.7-1.9 \mathrm{keV}$ and $2.1-2.3 \mathrm{keV}$ because of calibration uncertainties.

The source was also observed by the Hard X-ray Detector (HXD; Takahashi et al. 2007; Kokubun et al. 2007). The HXD/PIN data (the PIN array is sensitive between $\approx 15-60 \mathrm{keV}$ ) were reduced using standard tasks. The FTOOLS routine hxdpinxbpi is a pipeline task that first extracts spectral counts and corrects these for deadtime. Using the 'tuned' background model for the target observation provided by the Suzaku team as a starting point (Fukazawa et al. 2009), hxdpinxbpi also outputs a background spectrum including the cosmic X-ray background (CXB) component. However, after background subtraction, the residual source count rate was found to be $0.013 \mathrm{cts} \mathrm{s}^{-1}(15-60 \mathrm{keV})$, which is $\approx 5.0 \%$ of the gross count rate and similar to the level of background reproducibility for observations after 201228 We conservatively consider the source as a non-detection but note that fitting a PL to the detected net counts between 15 and $60 \mathrm{keV}$ returns an observed flux $F_{15-60} \approx 5 \times 10^{-12} \mathrm{erg} \mathrm{s}^{-1} \mathrm{~cm}^{-2}$, which is similar to the observed NuSTAR flux in the same band with the best fit models that we will discuss in the Results section.

NGC 7674 is also too faint to be detectable in the HXD/GSO array sensitive to much higher energies, and those data are not considered here.

\subsection{Swift}

The source has been observed by Swift (Gehrels et al. 2004) on 17 occasions 2011 onwards, with exposure times ranging over $\approx 470$ 5100 s using the X-Ray Telescope (XRT; Burrows et al. 2005) sensitive to photons between $\sim 0.3-10 \mathrm{keV}$. The individual observations are listed in the Appendix. We extracted source and background spectra, together with response files, using the standard XRT Data Analysis Software tools with HEASOFT. The version of XRTPIPELINE used was 0.13.0. Source counts were extracted within a 20 " radius aperture, and background was extracted from an off-source sky region. Upper limits (for detection significance less than $10^{-3}$ ) were estimated using the sosta command in the XIMAGE package.

We first analysed the spectra individually, but the source lies at the limit of detectability in these observations, yielding only weak detections for observations longer than $2 \mathrm{ks}$, and non-detections in other cases. Nevertheless, the individual observations (detections and limits) allow a first check for any strong variations with time. These fits are also described in the Appendix, and no significant variations are found.

We then extracted a coadded XRT spectrum by combining the event files of the individual observations. This yields a dataset with a total exposure time of $48.8 \mathrm{ks}$. The source is well detected in this combined exposure, with a net count rate of $7.9( \pm 0.4) \times 10^{-3} \mathrm{ct} \mathrm{s}^{-1}$ over the energy range of $0.5-10 \mathrm{keV}$.

The source is classified as a non-detection by the Burst Alert Telescope (BAT; Barthelmy et al. 2005) sensitive over 14-195 keV using standard analysis adopted for the 70-month all-sky survey (Baumgartner et al. 2013), and we do not consider the BAT data further in this work. We do note, however, that a custom analysis by Koss et al. (2013) finds a $4.2 \sigma$ detection at the position 28 www.astro.isas.jaxa.jp/suzaku/analysis/hxd/pinnxb/
tuned/140530bgdd.pdf of NGC 7674 with a flux $F_{14-195}=9.9_{-2.4}^{+5.1} \times 10^{-12} \mathrm{erg} \mathrm{s}^{-1} \mathrm{~cm}^{-2}$, consistent with that inferred from our NUSTAR analysis described later.

\subsection{Optical Spectroscopy}

In preparation for, and in support of, the NUSTAR observations, we also obtained optical spectroscopy of NGC 7674 using the Low Resolution Imaging Spectrometer (LRIS) on the Keck Telescope (Oke et al. 1995). The observations were carried out on 2014 June 25 through a $1{ }^{\prime \prime} .0$ wide slit, using both the blue $(600$ lines $\mathrm{mm}^{-1}$ ) grism and the red (400 lines $\mathrm{mm}^{-1}$ ) grating, with the D560 dichroic. The night was photometric, with seeing close to 0.7 .

The blue spectral region is dominated by strong emission lines with blueshifted components, as reported in many previous works (e.g. Feldman et al. 1982). The red spectral region contains the isolated $\mathrm{Ca}$ II absorption triplet, which can be used to estimate the central black hole mass. This estimate is presented in the Appendix. The instrumental resolution in the red spectral region was measured to be $7.4 \AA$ (FWHM) using arc lamp spectra, corresponding to a velocity resolution of $\sigma_{\text {instr. }}=107 \mathrm{~km} \mathrm{~s}^{-1}$ close to the observed wavelength of the $\mathrm{Ca}$ triplet feature.

\subsection{X-ray Spectral Analysis Methodology}

In this section, we start by checking for consistency of the data between the various missions, and then describe the details of the spectral analysis models.

\subsubsection{Basic characterisation}

Fig.1 shows the NuSTAR, Suzaku, and Swift data sets overplotted in count rate units, stretching over two decades in observed energy from $0.5-78 \mathrm{keV}$. The spectral shape approximately matches between the missions and instruments over common energy ranges. In particular, there appears to be a broad hump dominating the NUSTAR band above $10 \mathrm{keV}$ and a sharp emission feature around $6 \mathrm{keV}$. These are strongly reminiscent of reflection resulting from Compton scattering and neutral $\mathrm{Fe}$ fluorescence, a common characteristic of obscured AGN X-ray spectra. The hump extends down to $\sim 3 \mathrm{keV}$ in all data sets, below which a different component appears to dominate in both the Suzaku/XIS and Swift/XRT data with the spectrum rising and peaking around $1 \mathrm{keV}$ (this is related to the peak in the effective area, in the spectral units of Fig. 1.).

Fitting a PL to the continuum over the common energy range of 3-10 keV (after ignoring the range of $5.5-7 \mathrm{keV}$ around the neutral $\mathrm{Fe} \mathrm{K} \alpha$ line) simultaneously to all missions returns a photon index $\Gamma=0.73 \pm 0.11$ with an acceptable fit statistic of $\chi^{2} /$ dof $=99.9 / 92$. This is much harder than the typical intrinsic photon indices $(\langle\Gamma\rangle \sim 1.9)$ seen in AGN X-ray spectra (cf. Nandra et al. 1997; Mateos et al. 2005; Piconcelli et al. 2005) and is suggestive of heavy obscuration. Fitting the same model to the data from each mission separately, the observed $2-10 \mathrm{keV}$ fluxes span the range of $F_{2-10} \approx(7-14) \times 10^{-13} \mathrm{erg} \mathrm{s}^{-1} \mathrm{~cm}^{-2}$ between the missions (with the harder Swift photon index giving the highest flux), and are fully consistent with each other at $90 \%$ confidence.

We note a mild discrepancy in the Swift XRT data with respect to the other missions. When examining the individual photon indices in the above fit to each mission separately, we find $\Gamma_{\mathrm{XRT}}=-$ $0.68 \pm 1.26$. This is harder than the median $\Gamma$ value from the other 
missions at $90 \%$ confidence. But at $95 \%$ confidence, we find that all missions do agree. The cause of this slight mismatch is not clear but is unlikely to be related to differing aperture sizes used for extracting spectra for the different missions, because we expect the unresolved AGN alone to be the dominant contributor at these energies. Instead, at soft energies, one may expect spatially extended emission and larger differences, which we will discuss later. In any case, since this discrepancy is relatively mild, and since all other observations are fully consistent with each other, we consider a joint analysis of the NuSTAR, Suzaku XIS, and coadded Swift XRT data to be justified, and this is the approach we follow in the rest of this paper.

\subsubsection{Reflection models}

For the detailed spectral fits, we will be fitting X-ray reflection models over much of the hard X-ray energy range. There are several canonical models available for fitting heavily obscured AGN spectra in XSPEC. Traditionally, PEXRAV (Magdziarz \& Zdziarski 1995) and its successor PEXMON including fluorescence (Nandra et al. 2007) have been used to characterise reflection features. These assume a slab obscurer/reflector with an infinite optical depth, as may be expected in a standard geometrically-thin accretion disc. The incident source in $\mathrm{X}$ rays is associated with power law radiation $\left(\mathrm{PL}_{\mathrm{AGN}}\right)$ from a hot electron accretion disc corona. More recently, there are models which simulate X-ray processing in finite optical depth toroidal media which are more physically appropriate for obscured AGN. Murphy \& Yaqoob (2009) provide tabulated results of Monte Carlo simulations of an AGN illuminating a doughnut shaped torus with a fixed opening angle and covering factor of 0.5 (the MYTORUS model). Brightman \& Nandra (2011) assume, instead, a torus defined as a conical section of a sphere with variable opening angle and hence variable covering factor (the TORUS model). Both models assume Solar abundances and treat absorption, reflection and $\mathrm{Fe} \mathrm{K} \alpha$ fluorescence self-consistently. MYTORUS additionally allows the freedom to vary the parameters of the scatterer, the 1.o.s obscurer, and the fluorescer, decoupled from one another. Both torus models assume that there is no $e$-folding cut-off energy to $\mathrm{PL}_{\mathrm{AGN}}$. For consistency, we make the same assumption in PEXMON. All models assume a uniform gas density spatial distribution.

Since the geometry of the obscuring/reflecting medium is unknown, we will use all three geometries above and investigate the range in intrinsic properties that can satisfy the observations.

\subsubsection{Additional model components}

Fixed Galactic absorption (PHABS $\mathrm{Gal}_{\mathrm{G}}$ ) with $N_{\mathrm{H}}=4.27 \times 10^{20} \mathrm{~cm}^{-2} \quad$ (Dickey \& Lockman 1990) was included in all models. We also included constants to account for cross-calibration uncertainties between the various detectors and missions. We found that one or two APEC (Smith et al. 2001) components and a soft power law $\left(\mathrm{PL}_{\mathrm{soft}}\right)$ were required to represent the energy range below $\sim 2 \mathrm{keV}$ in some models. We emphasise that these components are meant to serve as parametrisations only. Previous works have shown that the origin of the soft X-ray photons in obscured AGN is a complex mixture of AGN photoionisation, starburst emission, and power law contributions from X-ray binaries, among various possible origins (e.g. Sako et al. 2000; Kinkhabwala et al. 2002; Cappi et al. 2006; Guainazzi \& Bianchi 2007). Separating these various possible components in NGC 7674 will require high spatial and spectral resolution observations with Chandra and Athena, respectively. Our main focus is the origin of the higher energy X-rays, so we use the APEC and PL $\mathrm{L}_{\text {soft }}$ components simply to ensure that the spectral fits over the soft regime are statistically acceptable. In addition, we may expect spatially extended soft emission to contribute in differing amounts to the Suzaku and Swift spectra because of the differing spectral extraction apertures tuned to the sizes of the respective telescope point spread functions. We account for this simply by allowing the APEC components to vary independently between these two missions. The only cross-check required in this regard is that the soft X-ray flux measured by Swift XRT (with the smaller aperture) should not exceed that observed in Suzaku XIS. This cross-check was applied post-fitting and found to hold for the best fits presented in the following sections.

With regard to other components, some models preferred several layers of absorption in addition to Galactic, as follows:

(i) Nuclear obscuration: All models with a transmission component required a thick nuclear absorber, which we associate with the classical compact torus having a l.o.s. column density ' $N_{\mathrm{H}}$ (nuc)' well above $10^{24} \mathrm{~cm}^{-2}$.

(ii) Host galaxy absorption: Most models preferred the inclusion of a weak absorber screening the soft thermal and power law components (denoted by ' $N_{\mathrm{H}}$ (host)' with values of a few times $10^{21} \mathrm{~cm}^{-2}$ ). This corresponds to weak host galaxy reddening and is consistent with optical reddening of the Narrow Line Region, as we discuss later.

(iii) Scattering screen: One alternative scenario that we will investigate requires an additional absorbed power law continuum component, which can potentially be attributed to scattering of the intrinsic $\mathrm{PL}_{\mathrm{AGN}}$ into the l.o.s. The scattered fraction is $f_{\mathrm{scatt}}$, i.e. $\mathrm{PL}_{\mathrm{scatt}}=f_{\mathrm{scatt}} \times \mathrm{PL}_{\mathrm{AGN}}$. Absorption is required for this component, but as we will discuss later, the data cannot distinguish between the two possibilities of (a) a screen that absorbs only the scattered power law, and (b) a screen that affects all the compact nuclear components (direct, reflected, as well as scattered), with both possibilities allowed for in our analysis. We follow the more generic case (b) above, and term this component ' $N_{\mathrm{H}}$ (scatt).' It shows intermediate column density values with $N_{\mathrm{H}}(\mathrm{scatt}) \sim 10^{23} \mathrm{~cm}^{-2}$.

Finally, we found that inclusion of a Hydrogen-like Fe XXVI line at $6.97 \mathrm{keV}$, probably related to the ionised scattered continuum, provided a significant improvement in all models.

Our model values at any energy $E$ can most generically be described as follows.

$$
\begin{gathered}
F(E)=C \quad C e^{-\tau(\text { Gal })} e^{-\tau(\text { host })}\left[\operatorname{APEC}(\mathrm{s})+\mathrm{PL}_{\mathrm{soft}}\right. \\
\left.+e^{-\tau(\text { scatt })}\left[\operatorname{Torus}\left(\Gamma, N_{\mathrm{H}}(\text { nuc })\right)+\operatorname{PL}_{\mathrm{scatt}}(\Gamma)+\text { Line }\right]\right],
\end{gathered}
$$

where 'Torus' represents one of our three primary models including either (i) PEXMON (Model P), or (ii) Brightman \& Nandra TORUS (Model T), or (iii) Murphy \& Yaqoob MYTORUs (Model M), in order to model the primary nuclear obscurer and reflector with column density $N_{\mathrm{H}}$ (nuc). ' $C$ ' represents cross-calibration constants, $\tau$ is the optical depth $N_{\mathrm{H}} \times \sigma\left(E_{z}\right)$ at the rest-frame energy $E_{z}$ of the absorber, and 'Line' refers to the Fe XXVI emission line. We emphasise that not all models require the complexity implied by the above generic description of components. 


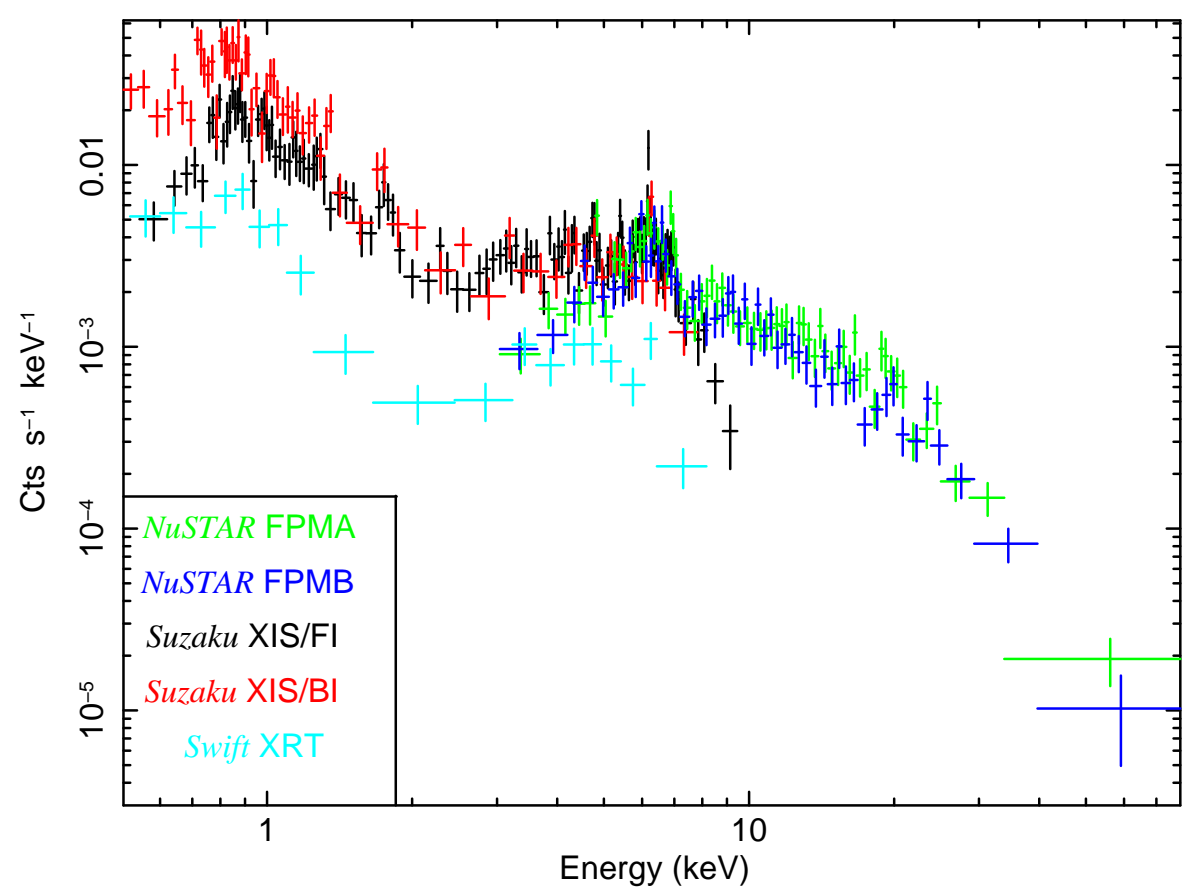

Figure 1. The NuSTAR, Suzaku and Swift data plotted in observed count rate units.

\section{RESULTS}

\subsection{Model P: PEXMON}

We began with fitting Model P (PEXMON). This slab reflection model can fit the Compton hump successfully but strongly overpredicts the $\mathrm{Fe} \mathrm{K} \alpha$ line strength when using fixed abundances at the Solar value. Letting the elemental abundance $\left(A=A_{\mathrm{Fe}}\right)$ vary freely yields an acceptable solution $\left(\chi^{2} / \mathrm{dof}=324.7 / 305\right)$ with $\Gamma=2.1_{-0.1}^{+0.2}$, $A=0.5 \pm 0.1$ and a slab inclination of at least $77 \mathrm{deg}$. The inclination affects the 'peakiness' of the Compton hump, with lower inclinations being too 'peaky' as compared to the data 29 This model $\mathrm{P}$ solution is plotted in Fig. 2 and fit parameters are listed in Table_2 The bottom panel in the same figure demonstrates the strong $\mathrm{Fe} \mathrm{K} \alpha$ residuals with $A$ fixed to Solar and $\Gamma=2.1$ (fixed to the same value as the canonical model $\mathrm{P}$ ); letting $\Gamma$ vary freely did not provide a better fit than that stated in Table 2

A transmitted component of the direct AGN power law ( $\mathrm{PL}_{\mathrm{AGN}}$ ) is included in this model. Compton scattering and photoelectric absorption by the torus are simulated with standard multiplicative models CABS and ZPHABS, respectively, with the gas column density $N_{\mathrm{H}}$ (nuc) tied between the two models. The best-fit $N_{\mathrm{H}}$ (nuc) $=3.4_{-0.6}^{+0.8} \times 10^{24} \mathrm{~cm}^{-2}$. The reflection component dominates the absorbed transmitted component over the entire spectral range probed, i.e. the source is fully reflection-dominated. The excess of the reflection component is a factor of $\sim 3$ around $30 \mathrm{keV}$. We note that excluding the transmission component results in a moderate increase in the fit statistic to $\chi^{2} / \mathrm{dof}=334.5 / 306$, which is only marginally significant at the $3 \sigma$ level. In other words, a transmission component is not strongly required.

We find that the cross-calibration constants between the various missions are consistent with 1 within the uncertainties. The strength of the FeXXVI line is $\mathrm{EW}(\mathrm{Fe} X X V I) \approx 200 \mathrm{eV}$. At the

29 For an illustration of this effect, see Fig. 5 of Magdziarz \& Zdziarski (1995). soft end, the Suzaku XIS data require two APEC components with temperatures $k T \approx 0.1$ and $0.6 \mathrm{keV}$, whereas the Swift XRT data probing smaller apertures require only a single lower temperature component. The APEC abundances are $\sim 0.01-0.2$. Multiple thermal models with widely differing temperatures and abundances are quite common in luminous infrared galaxies (e.g. Ranalli et al. 2008) and have also been seen in other CTAGN (e.g. Konami et al. 2012). There is, however, strong degeneracy between the abundances and temperatures in data with low spatial and spectral resolution, and tieing all the Suzaku and Swift model abundances to each other also yields an acceptable solution $\left(\chi^{2} / \mathrm{dof}=332 / 307\right)$ with a global abundance $A=0.27_{-0.14}^{+0.56}$, a Swift $\mathrm{APEC}_{3}$ component temperature of $k T_{3}=0.19_{-0.08}^{+0.03} \mathrm{keV}$, and no significant changes to the corresponding temperatures for the $\mathrm{APEC}_{1}$ and $\mathrm{APEC}_{2}$ Suzaku components. However, we emphasise that we are not attempting to constrain the origin of the soft X-ray emission in detail here.

The best fit model needs only one additional layer of absorbing column density $N_{\mathrm{H}}$ (host) $=4.0_{-1.6}^{+2.1} \times 10^{21} \mathrm{~cm}^{-2}$. This is a rather thin screen and corresponds to an optical extinction $E_{\mathrm{B}-\mathrm{V}}=0.7_{-0.3}^{+0.4} \mathrm{mag}$ for a standard Galactic gas-to-dust ratio (Bohlin et al. 1978). This is consistent with the reddening expected from the Balmer decrement of 4.80 (Bassani et al. 1999). Removing this layer significantly worsens the fit to $\chi^{2} / \mathrm{dof}=342.1 / 306$. But most of the change is concentrated at the softest energies (resulting in an increased APEC temperature) which we are not modelling in detail; the primary AGN reflection component is unaffected.

\subsection{Model T: TORUS}

PEXMON assumes a slab geometry with infinite density, which is unlikely to be representative of the toroidal obscurer envisaged by AGN unification schemes. So we next turned to the Brightman \& Nandra TORUS model. This also allows us to explore alternatives to our low $A_{\mathrm{Fe}}$ solution, because abundances are 


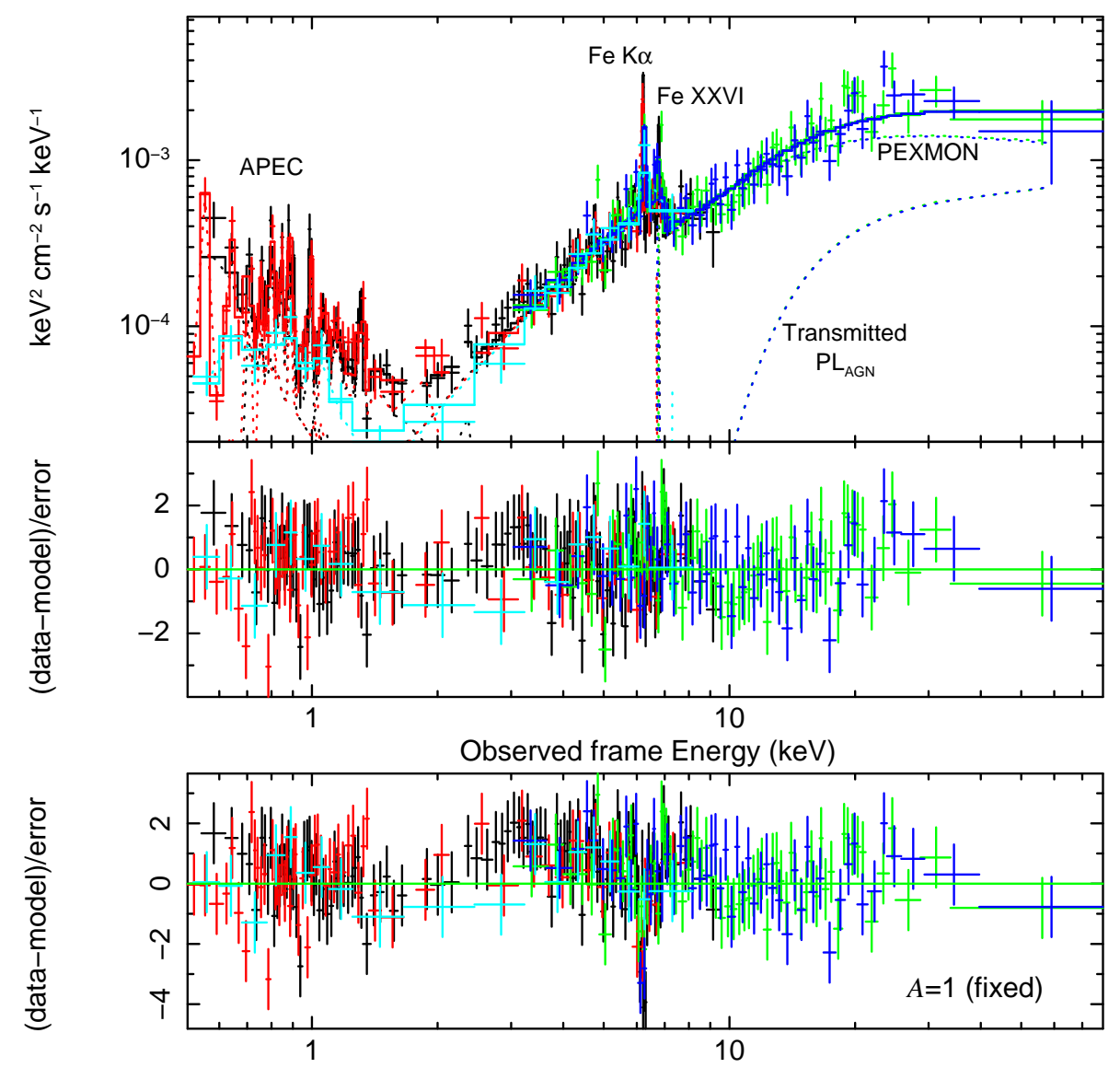

Figure 2. PEXMON model fit to the Suzaku XIS FI (black), XIS BI (red), NuSTAR FPMA (green), FPMB (blue) and Swift XRT (cyan) data. The central panel shows the fit residuals. The Fe abundance for this fit is close to 0.5 (Table 2). The bottom panel shows the residuals to a fit with fixed abundance $A=1$. Notice the strong over-prediction at the neutral Fe K $\alpha$ energy.

fixed at Solar values in this model. In this case, an acceptable solution may be found if we effectively dilute the continuum emission from the torus at the $\mathrm{Fe} \mathrm{K} \alpha$ line energy with a stronger scattered component $\left(\mathrm{PL}_{\mathrm{scatt}}\right) 30$ Such a solution is plotted in Fig. 3 where $N_{\mathrm{H}}$ (nuc) is above $10^{25} \mathrm{~cm}^{-2}$, well within the Comptonthick regime. The scattered fraction is $f_{\text {scatt }} \approx 4 \%$, which raises the continuum at the $\mathrm{Fe} \mathrm{K} \alpha$ line energy sufficiently to produce a fully acceptable fit $\left(\chi^{2} / \mathrm{dof}=320.2 / 300\right)$. For this fit, we froze the inclination angle $\theta_{\text {inc }}$ of the torus to be close to edge-on, as is recommended for exploration of the full range of possible covering factors (Brightman et al. 2015). There are uncertainties in the TORUS model at edge-on inclinations, as discussed by Liu \& Li (2015). We checked that varying $\theta_{\text {inc }}$ did not affect our final inferences. The best fit opening angle is $\theta_{\text {tor }}=62_{-11}^{+15} \mathrm{deg}$. Smaller opening angles produce a worse fit because they result in Compton humps peakier than required by the data 31 Large values of $\theta_{\text {tor }}$ are allowed up to $\theta_{\text {tor }}=77 \mathrm{deg}$, beyond which the torus effectively becomes too geometrically thin to produce a sufficiently strong Compton hump.

In this model, however, an additional intermediate column

30 See the presentation by T. Yaboob at http://cxc.cfa.harvard.edu/ChandraDecade/ for more discussions of such a warm scatterer scenario.

31 see Fig. 1 of Brightman et al. (2015) for an illustration of the effect of changing $\theta_{\text {tor }}$. obscurer $N_{\mathrm{H}}$ (scatt) $\sim 10^{23} \mathrm{~cm}^{-2}$ is required, so as not to overproduce the soft X-ray flux. We note that in our model configuration, $N_{\mathrm{H}}$ (scatt) affects $\mathrm{PL}_{\text {scatt }}$ as well as the compact nuclear TORUS component. But modifying the model such that $N_{\mathrm{H}}$ (scatt) affects PL(scatt) alone also yields a fully acceptable solution with $\chi^{2} / \mathrm{dof}=323.3 / 300$. This consistency results from the fact that $\mathrm{PL}_{\text {scatt }}$ completely dominates over the TORUS reflection continuum at energies of around a few $\mathrm{keV}$ - energies below which gas with an intermediate column density of $\sim 10^{23} \mathrm{~cm}^{-2}$ is an effective absorber (cf. Fig. 3).

This above $N_{\mathrm{H}}$ (scatt) column is $\approx 20$ times higher than the host galaxy absorbing layer $\left(N_{\mathrm{H}}\right.$ (host)) discussed in the previous sub-section, and would imply correspondingly higher optical reddening. So how viable is this? NGC 7674 is a known luminous infrared galaxy with ongoing star formation and physical interactions with its neighbouring galaxies - all of which could result in obscuring matter being strewn around the host galaxy on multiple scales. Other examples of nearby galaxies with complex multiple layers of absorption identified by NUSTAR include NGC 7582 (Rivers et al. 2015) and IC 751 (Ricci et al. 2016). Therefore, the presence of an additional absorber in the innermost parts of the host galaxy which obscures the emergent flux from the nuclear regions is not implausible.

Detailed spatially resolved studies of the nuclear region so far neither require, nor rule out, such a screen. For example, the study of Fischer et al. (2013) does not show an obvious [O III] flux decrement at the nucleus, but the spatial resolution of their spectral sam- 
pling is $\approx 30 \mathrm{pc}$, much larger than the typical sizes of compact tori, and may potentially allow for additional absorbers below the resolution limit. Alternatively, the outflowing gas associated with the powerful nuclear outflow known in NGC 7674 may also serve as a screening medium for X-rays.

In summary, the introduction of $N_{\mathrm{H}}$ (scatt) is clearly somewhat ad hoc and is motivated by the adopted scattering scenario in a fixed Solar metallicity torus model. Nevertheless, we cannot rule it out based upon the present evidence, and we also note that the presence

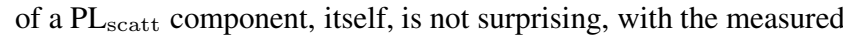
$f_{\text {scatt }}$ value of a few per cent being fully consistent with that seen in other nearby AGN (e.g. Cappi et al. 2006). We have introduced this only as one possible scenario, and as we will discuss in the following section, more complex models could alleviate the need for this screen altogether.

The soft energies can be fitted in a very similar fashion to Model P, with one difference being the presence of a significant, but faint, $\mathrm{PL}_{\text {soft }}$ component. $\mathrm{PL}_{\text {soft }}$ dominates only in a very narrow energy range around $2 \mathrm{keV}$, as a result of which we found that its photon index needed to be fixed, otherwise the fit attempted a very hard slope with contribution to the highest energy NuSTAR range. This component is not required in model $\mathrm{P}$ because the reflected component is much more prominent in that case, leaving no deficit around $2 \mathrm{keV}$ (cf. Fig.2). Since the purpose of this component is to account for the soft energies, we fixed $\Gamma_{\text {soft }}=2.0$, similar to the spectral slope of the emission from X-ray binaries (e.g. Ranalli et al.2003). We also checked that allowing small variations of $\Delta \Gamma_{\text {soft }}= \pm 0.3$ do not affect the modelling of the main AGN component.

\subsection{Model M: MYTORUS}

We first fitted a standard 'coupled' MYTORUS model here with all parameters between the 1.o.s. and toroidal scattering components tied to each other. This solution is very similar in essence to model $\mathrm{T}$, and the fit statistic of $\chi^{2} / \mathrm{dof}=325 / 300$ is compatible with both previous models. Quantitatively, models $\mathrm{T}$ and $\mathrm{M}$ differ in that the derived equatorial column density through the torus $\left(N_{\mathrm{H}}(\mathrm{eq})\right)$ is pegged at the allowed upper model threshold of $10^{25} \mathrm{~cm}^{-2}$ in model M. Similarly, $\theta_{\text {inc }}$ is just above (and very close to) the allowed lower model threshold of $\theta_{\text {inc }}=60^{\circ}$. Such parameter pegging has been seen in other objects also, and reflects the constraint of the assumed doughnut geometry in this model as a result of which the value of $N_{\mathrm{H}}$ (nuc) is directly coupled to $\theta_{\text {inc }}$ (e.g., Gandhi et al. 2014; Baloković et al. 2014; Lansbury et al. 2015). Smaller inclination angles are not obscured by the torus in this model, whereas higher $\theta_{\text {inc }}$ values produce a Compton hump which is too peaky relative to the data. Additional model components are also very similar to model T. The fitted parameters of this model are listed in Table 2 and the solution is shown in Appendix figure A1.

\subsubsection{Decoupled MYTORUS model}

We next tried several versions of the more complex 'decoupled' mode in MYTORUS. In this mode, the direct 1.o.s. absorption, the toroidal Compton scattering emission, and the fluorescence emission components are not necessarily coupled to each other. By varying the $N_{\mathrm{H}}$, the inclination angles or the relative normalisations associated with these components, one can effectively simulate a variety of scenarios including a clumpy obscurer or varying elemental abundances (for example). This may also potentially allow us to remove the need for the additional ' $N_{\mathrm{H}}$ (scatt)' layer that was introduced in Section 3.2 for absorbing the scattered power law that dilutes $\mathrm{EW}(\mathrm{Fe} \mathrm{K} \alpha)$.

We first confirmed that simply decoupling the normalisation constant of the fluorescence line $A_{L}$ from the normalisation of the Compton scattering component $A_{S}$ allowed a very good fit (with a fitted sub-unity value of $A_{L}=0.26 \pm 0.06$ ) with no extra $\mathrm{PL}_{\text {scatt }}$ component required, equivalent to model $\mathrm{P}$. The problem is that such a decoupling cannot be self-consistently interpreted as having sub-Solar abundances, because of the assumption of Solar abundances for the Compton scattering component that produces the overall continuum shape of the Compton hump.

We also attempted more complex scenarios, including the possibility of having multiple scatterers. Such a scenario includes two components inclined at orthogonal angles of 0 [face-on] and at 90 deg [edge-on] with $A_{S}=A_{L}$ for each component, and is discussed at length by Yaqoob (2012). But these models appeared to require extreme decoupling between the two scatterers. For instance, allowing freely varying cross-normalisation constants between these scatterers, the face-on reflection component $\left(A_{S 0}\right.$ in Yaboob's terminology) prefers a normalisation $\gtrsim 5$ times stronger than the edge-on component $\left(A_{S 90}\right)$ responsible for the 1.o.s. obscuration. This implies a strong departure from the default timesteady illuminated MYTORUS geometry with covering factor of 0.5 . Such a scenario with strong variability on characteristic timescales relevant to the inner torus is discussed and argued against in Section 4.2.3

Alternatively, the column densities between the two scatterers may also be untied. This is equivalent to simulating a clumpy medium with the line-of-sight obscuration differing from global. Such a solution is shown in Fig. 4 The spectrum can be crudely fit $\left(\chi^{2} /\right.$ dof $\left.=371 / 301\right)$ with a low 1.o.s column associated with the edge on obscurer $N_{\mathrm{H}}($ nuc $)=1.3( \pm 0.3) \times 10^{23} \mathrm{~cm}^{-2}$, This component is denoted by 'MYT(Z90)' in the figure, as in Yaqoob (2012). However, this also requires a very hard photon index for $\mathrm{PL}_{\mathrm{AGN}}$, with $\Gamma=1.40_{-u}^{+0.08}$ pegged at the lower limit allowed by the model. The out-of-sight global column from the face-on scatterer is $N_{\mathrm{H}}(\mathrm{S} 0)=2.4_{-0.8}^{+1.7} \times 10^{24} \mathrm{~cm}^{-2}$ (with corresponding Compton scattered and fluorescence components denoted by 'MYT(S0)' and 'MYT(L0)'; Ibid.). There is more than an order of magnitude difference between the two column densities. In order to prevent the strong normalisation departure mentioned in the preceding paragraph, we limited $A_{S 0}$ to a maximum value of 1.20 , and the fit does, indeed, want to exceed this limit, with $A_{S 0}$ pegged at $1.20_{-0.07}^{+u}$. The scattered fraction is high, with $f_{\text {scatt }}=0.12_{-0.06}^{+0.04}$ but we stress that there is no additional ad hoc screen associated with the aforementioned $N_{\mathrm{H}}$ (scatt) component in this decoupled model (or in any of the others discussed in this section). The overall fit statistic is much worse $\left(\Delta \chi^{2}=+46\right.$ for a single extra dof) than the default model $\mathrm{M}$ solution shown in Table 2

Scenarios with strong clumpiness have also been discussed for Mrk 3 (Yaqoob et al. 2015; Guainazzi et al. 2016). But such a scenario is not obviously applicable to NGC 7674. Mrk 3 is known to be continuously variable in flux and line-of-sight column density, whereas NGC 7674 has been stable since the BeppoSAX observations in 1996. Furthermore, it is clear from the discussion of the pegged photon index and normalisation values above that the fit is attempting to converge on a harder, more reflection-dominated continuum shape, suggesting that it does prefer a higher line-of-sight column density.

Unless the direct PL $_{\mathrm{AGN}}$ component can be robustly detected, such solutions are highly complex and degenerate, and do not yield 
any obvious physically useful insights on the nuclear medium beyond our three canonical models discussed in Table 2 This is particularly true for reflection-dominated AGN with no direct detection of PLAGN (e.g., see detailed discussion in Yaqoob 2012 on this point). Therefore, although we cannot rule out more complex models at this stage, we do not investigate the decoupled modes in greater detail herein. Future high signal-to-noise broadband spectra, and robust detections of reflection features such as the fluorescence Compton shoulder with X-ray calorimeters, could yield more insight on such models.

\section{DISCUSSION}

\subsection{Intrinsic luminosity}

Our three models return intrinsic $L_{2-10}$ values ranging over $\approx(3-$ 5) $\times 10^{43} \mathrm{erg} \mathrm{s}^{-1}$ for the best fit parameters in Table 2 The narrow range of these luminosities is noteworthy, despite the differing geometries inherent to these models. Placing this range in the context of other well-known CTAGN, the luminosity of NGC 7674 is similar to NGC 1068, and is factor of $\approx 2$ lower than Mrk 34 (Bauer et al. 2014; Gandhi et al. 2014). The former object is the prototypical reflection-dominated AGN, while the latter is the most luminous known bona fide CTAGN within $\sim 250 \mathrm{Mpc}$. For the observed (i.e. absorbed) spectra, we have $F_{2-10}^{\text {obs }}=7.7 \times 10^{-13} \mathrm{erg} \mathrm{s}^{-1} \mathrm{~cm}^{-2}$, or $L_{2-10}^{\text {obs }}=1.5 \times 10^{42} \mathrm{erg} \mathrm{s}^{-1}$ - a factor of $\approx 20-30$ times lower than the inferred intrinsic power.

In order to estimate realistic uncertainties on the intrinsic luminosities, we stepped over a 2-dimensional grid of $\Gamma$ and normalisation $(N)$ for the intrinsic PL $_{\mathrm{AGN}}$ - the two parameters which determine the absorption-corrected flux (and hence direct luminosity). Carrying out fits over the grid yields a $\chi^{2}$ value for each combination of $\Gamma$ and $N$, and thus effectively for each value of $L_{2-10}$. Different combinations of the two starting parameters can return identical $L_{2-10}$ values, so the 1-dimensional space of $\chi^{2}$ as a function of $L_{2-10}$ is not unique. But the overall uncertainties can be gauged from the envelope of $\chi^{2}$ contours for all combinations. This envelope is plotted in Fig.5 for both models $\mathrm{M}$ and T. The figure shows that realistic $L_{2-10}$ uncertainties span the range of $\approx(1.3-$ 13) $\times 10^{43} \mathrm{erg} \mathrm{s}^{-1}$, or about an order of magnitude.

Our estimate of the mass of the supermassive black hole (SMBH) in NGC 7674 is $M_{\mathrm{BH}}<10^{7.43} \mathrm{M}_{\odot}$ (see Appendix). Using our best-fit $L_{2-10}$ range of $(3-5) \times 10^{43} \mathrm{erg} \mathrm{s}^{-1}$ together with a bolometric correction likely range of $L_{\mathrm{Bol}} / L_{2-10} \approx 10-20$ Vasudevan \& Fabian 2007), we estimate an Eddington ratio range of $L_{\mathrm{Bol}} / L_{\mathrm{Edd}}>0.09-0.29$. Including the full range of $\mathrm{X}$-ray luminosity uncertainties expands this range to $L_{\mathrm{Bol}} / L_{\mathrm{Edd}}>0.04-0.74$, implying the presence of an efficiently accreting AGN.

\subsubsection{Multiwavelength comparisons}

Multiwavelength scaling relations are very useful for comparison of intrinsic luminosity estimates, especially in the heavily Compton-thick regime where the direct X-ray emission is not detected. Two commonly used multiwavelength indicators of intrinsic AGN power are the mid-infrared continuum and the optical forbidden emission line luminosities, in particular the [O III] 15007 ̊ line doublet (e.g. Gandhi et al. 2009; Lamastra et al. 2009, and references therein). We compare NGC 7674 to these relations here.

NGC 7674 has been observed at high angular resolution in the mid-infrared using $8 \mathrm{~m}$ class telescopes. It is found to have a $12 \mu \mathrm{m}$ infrared luminosity of $L_{12}=1.8( \pm 0.3) \times 10^{44} \mathrm{erg} \mathrm{s}^{-1}$ (Asmus et al. 2014). Using this value of $L_{12}$, the relation between infrared and X-ray luminosities for local AGN (Asmus et al. 2015; Gandhi et al. 2009) predicts an intrinsic $L_{2-10}$ range of (6$9) \times 10^{43} \mathrm{erg} \mathrm{s}^{-1}$ at $68 \%$ confidence, which is marginally higher than the mean best fit luminosities from our spectral analysis, but entirely consistent with the full confidence regions for both models shown in Fig.5 Assuming a $6 \mu \mathrm{m}$ luminosity lower by a factor of 2 as compared to $L_{12}$ (cf. Goulding et al. 2012) together with the $L_{6} / L_{2-10}$ relation by Stern (2015) relevant for high luminosity AGN, the predicted $L_{2-10}$ decreases by a further 0.1 dex. The angular resolution of the mid-infrared observations used (seeing-limited at $\approx 0.4 \mathrm{arcsec}$ ) corresponds to a physical scale of $\approx 0.24 \mathrm{kpc}$ for the unresolved nuclear emission at the distance of NGC 7674, and represents the best direct measure of the intrinsic AGN power, with emission from surrounding star formation being largely resolved out. A further check on any contamination by non-AGN components may be obtained from the midinfrared colour of NGC 7674 as tabulated in the WISE/AllWISE catalogue (Wright et al. 2010; Cutri et al. 2013). The $W 1-W 2$ colour is $1.16 \pm 0.03 \mathrm{mag}$, which places the source above the colour threshold of $W 1-W 2>0.8$ identified by Stern et al. (2012) where the mid-infrared emission is likely to be AGN-dominated.

Comparing next to the [O III] emission line, Bassani et al. (1999) present the [O III] 15007 luminosity of the source as $L_{[\mathrm{O} \mathrm{III}]}=3.5( \pm 0.2) \times 10^{42} \mathrm{erg} \mathrm{s}^{-1}$. This value is derived after correcting for dust reddening based upon a Balmer decrement of 4.80 . Using the $L_{2-10} / L_{[\mathrm{O} \text { III }]}$ relationship presented in Lamastra et al. (2009) with a scatter of $0.6 \mathrm{dex}$, we expect $L_{2-10}=5_{-4}^{+7} \times 10^{43} \mathrm{erg} \mathrm{s}^{-1}$ at $68 \%$ confidence, overlapping well with our spectrally modelled intrinsic luminosity range.

In summary, the best fit X-ray luminosity measured from our spectral analysis agrees well with the multiwavelength comparisons above, especially when considering the full range of realistic uncertainties on the spectral modelling (Fig.5). This is encouraging given that this source appears to be heavily Compton-thick and that the true torus geometry is unknown. Broadband spectral modelling of high signal-to-noise X-ray data is what enables us to get such good agreement.

\subsubsection{The soft X-ray component}

The absorption-corrected luminosity in the XIS $0.5-2 \mathrm{keV}$ band is $L_{0.5-2}^{\mathrm{APEC}} \approx 4.5 \times 10^{42} \mathrm{erg} \mathrm{s}^{-1}$. This is for both APEC components combined, but is dominated by a factor of 4 by the lower temperature component. Using the relation for star-forming galaxies by Mineo et al. (2012) between the thermal component luminosity and the star formation rate (SFR), we estimate an X-ray derived $\mathrm{SFR}_{\mathrm{X}-\text { ray }} \approx 9 \times 10^{3} \mathrm{M}_{\odot} \mathrm{yr}^{-1}$.

This may be compared to the infrared derived SFR, based upon the far-infrared continuum luminosity $L_{\mathrm{IR}}$ and its relation to $\mathrm{SFR}_{\mathrm{IR}}$ (Kennicutt 1998). For NGC 7674, $L_{\mathrm{IR}} \approx 10^{11.56} \mathrm{~L}_{\odot}$ (Koss et al. 2013), which yields $\mathrm{SFR}_{\mathrm{IR}} \approx 60 \mathrm{M}_{\odot} \mathrm{yr}^{-1}$. This is more than two orders of magnitude lower than the estimated SFR $_{\mathrm{X}-\text { ray }}$, and implies that starburst-powered APEC components alone are an unphysical representation of the soft X-ray emission in NGC 7674. Photoionisation could instead power some of this, as we have already alluded to on several occasions. We also note that uncertainties related to absorption correction of soft X-rays cannot account for the extremely high $\mathrm{SFR}_{\mathrm{X}-\text { ray }}$. Ignoring absorption corrections and using the observed (i.e. absorbed) $L_{0.5-2}^{\mathrm{APC}}$ directly still results in $\mathrm{SFR}_{\mathrm{X}-\text { ray }} \approx 950 \mathrm{M}_{\odot} \mathrm{yr}^{-1}$, far higher than $\mathrm{SFR}_{\mathrm{IR}}$. 
Table 2. Results of X-ray spectral fitting to NGC 7674

\begin{tabular}{|c|c|c|c|c|c|}
\hline Component & Parameter & Model P & Model T & Model M & Units \\
\hline \multirow[t]{8}{*}{ Primary Absorber/Reflector } & $N_{\mathrm{H}}($ nuc $)$ & $3.4_{-0.6}^{+0.8}$ & $36_{-10}^{+u}$ & $5.5_{-2.3}^{+u}$ & $10^{24} \mathrm{~cm}^{-2}$ \\
\hline & $N_{\mathrm{H}}(\mathrm{eq})$ & 11 & 11 & $10.0_{-4.1}^{+u}$ & $10^{24} \mathrm{~cm}^{-2}$ \\
\hline & $\theta_{\text {inc }}$ & $85_{-8}^{+u}$ & $87.1^{f}$ & $65_{-4}^{+9.1}$ & deg \\
\hline & $\theta_{\text {tor }}$ & - & $62_{-11}^{+15}$ & - & deg \\
\hline & $R$ & $-1^{f}$ & - & - & \\
\hline & $A_{\mathrm{Fe}}$ & $0.51_{-0.11}^{+0.10}$ & $\_\ddagger$ & $\_\ddagger$ & \\
\hline & $\mathrm{EW}(\mathrm{Fe} \mathrm{K} \alpha)$ & $\ldots \ldots \ldots \ldots$ & $0.38_{-0.09}^{+0.10} \ldots$ & $\ldots \ldots \ldots . . .^{\dagger}$ & $\mathrm{keV}$ \\
\hline & $\mathrm{EW}(\mathrm{Fe} X X \mathrm{XI})$ & & $0.20_{-0.09}^{+0.09}$ & $\ldots \ldots \ldots . .^{\dagger}$ & $\mathrm{keV}$ \\
\hline \multirow[t]{2}{*}{$\mathrm{PL}_{\mathrm{AGN}}$} & $\Gamma$ & $2.07_{-0.11}^{+0.15}$ & $1.80_{-0.11}^{+0.15}$ & $1.93_{-0.12}^{+0.28}$ & \\
\hline & & \multicolumn{3}{|c|}{ Additional components } & \\
\hline \multirow{3}{*}{$\begin{array}{l}\text { Host absorption } \\
\operatorname{APEC}_{1}^{a}\end{array}$} & $N_{\mathrm{H}}($ host $)$ & $4.0_{-1.6}^{+2.1}$ & $3.1_{-1.1}^{+1.7}$ & $3.4_{-0.2}^{+0.2}$ & $10^{21} \mathrm{~cm}^{-2}$ \\
\hline & $k T_{\text {apec }_{1}}$ & $\begin{array}{l}0.11_{-0.02}^{+0.603} \\
+0.03\end{array}$ & $0.12_{-0.03}^{+0.1}$ & $0.11_{-0.04}^{+0.04}$ & $\mathrm{keV}$ \\
\hline & $A_{\text {apec }_{1}}$ & $0.1_{-0.08}^{+u}$ & $0.1_{-0.09}^{+0.60}$ & $0.1_{-0.07}^{+0.84}$ & \\
\hline \multirow[t]{2}{*}{$\operatorname{APEC}_{2}^{a}$} & $k T_{\text {apec }_{2}}$ & $0.56_{-0.07}^{+0.07}$ & $0.59_{-0.07}^{+0.07}$ & $0.59_{-0.08}^{+0.09}$ & $\mathrm{keV}$ \\
\hline & $A_{\mathrm{apec}_{2}}$ & $0.3_{-0.1}^{+0.8}$ & $0.2_{-0.1}^{+0.5}$ & $0.3_{-0.2}^{+0.5}$ & \\
\hline \multirow[t]{2}{*}{$\operatorname{APEC}_{3}^{a}$} & $k T_{\text {apec }_{3}}$ & $0.17_{-0.04}^{+0.05}$ & $0.15_{-0.04}^{+0.06}$ & $0.14_{-0.04}^{+0.06}$ & $\mathrm{keV}$ \\
\hline & $A_{\text {apec }_{3}}$ & $0.01_{-u}^{+0.07}$ & $0.01_{-u}^{+0.04}$ & $0.01_{-u}^{+0.16}$ & \\
\hline \multirow[t]{2}{*}{$\mathrm{PL}_{\text {soft }}$} & $\Gamma_{\text {soft }}$ & $-u$ & $2.0^{f}$ & $2.0^{f}$ & \\
\hline & Norm & - & $2.5_{-1.5}^{+1.2}$ & $2.5_{-1.5}^{+1.4}$ & $10^{-5} \mathrm{ph} \mathrm{keV}^{-1} \mathrm{~cm}^{-2} \mathrm{~s}^{-1}$ \\
\hline \multirow[t]{2}{*}{ Scattering } & $f_{\text {scatt }}$ & - & $3.5 \pm 1.2$ & $6.1_{-3.1}^{+3.6}$ & $10^{-2}$ \\
\hline & $N_{\mathrm{H}}($ scatt $)$ & - & $9.4 \pm 2.5$ & $10.6_{-2.3}^{+3.3}$ & $10^{22} \mathrm{~cm}^{-2}$ \\
\hline$C_{\mathrm{XIS}}^{\mathrm{XIS} \text { BI }}$ cross-calib & CONST & $1.00 \pm 0.07$ & $1.01 \pm 0.07$ & $1.00_{-0.07}^{+0.07}$ & \\
\hline$C_{\mathrm{XIS} F \mathrm{~F}}^{\mathrm{FPMA}}$ cross-calib & CONST & $1.06_{-0.07}^{+0.08}$ & $1.11 \pm 0.08$ & $1.12_{-0.08}^{+0.09}$ & \\
\hline$C_{\text {XIS FI }}^{\text {FPMB }}$ cross-calib & CONST & $1.04_{-0.07}^{+0.09}$ & $1.10_{-0.08}^{+0.05}$ & $1.11_{-0.09}^{+0.09}$ & \\
\hline$C_{\mathrm{XISFI}}^{\mathrm{XRT}}$ cross-calib & CONST & $0.98_{-0.13}^{+0.13}$ & $1.01 \pm 0.14$ & $1.00_{-0.14}^{+0.15}$ & \\
\hline$\chi^{2} / \mathrm{dof}$ & & $325 / 305$ & $320 / 300$ & $325 / 300$ & \\
\hline
\end{tabular}

Model P: PEXMON component fit (Nandra et al. 2007).

Model M: MYTORUS coupled component fit (Murphy \& Yaqoob 2009).

Model T: TORUS model component fit (Brightman \& Nandra 2011).

${ }^{u}$ unconstrained to within the model limits. $N_{\mathrm{H}}$ upper limits are $10^{25} \mathrm{~cm}^{-2}$ and $10^{26} \mathrm{~cm}^{-2}$ for MYTORUS and TORUS, respectively. APEC is defined between abundances of 0 to 5 , relative to Solar.

$f_{\text {fixed. }}$

${ }^{\dagger}$ Equivalent width measured using a simple Gaussian atop a locally fitted powerlaw continuum.

${ }^{a} \mathrm{APEC}_{1}$ and $\mathrm{APEC}_{2}$ represent thermal components in Suzaku XIS, and $\mathrm{APEC}_{3}$ is for Swift XRT.

${ }^{\ddagger}$ These models are defined for abundances fixed to Solar only.

We note, however, that the estimated value of SFR $I R$ is itself large. For example, it is about 10 times above the SFR of the prototypical starburst galaxy M82 (e.g. Telesco \& Harper 1980). Such high star formation is likely to power extended ionised gas emission, and we will return to possible implications of this in Section 4.3 .3

\subsection{The nature of the long-term flux changes}

Fig.6 shows the long-term X-ray light curve of NGC 7674 over a period of about 37 years. As first noted by Bianchi et al. (2005), the source showed a decline by a factor of $\sim 3$ in the $2-10 \mathrm{keV}$ band between the first detection by HEAO in the late 1970s and the Ginga measurement in 1989, followed by a further order of magnitude flux decrease in 1996 when BeppoSAX identified the source as a CTAGN (Malaguti et al. 1998). Thereafter, the source flux has remained constant with no significant spectral or flux variation for the past $\approx 20$ years. This now includes the most recent Suzaku and NuSTAR observations. The individual Swift observations spanning the period of 2011-2014 detailed in the Appendix also show fluxes or detection limits broadly consistent with BeppoSAX, Suzaku and NuSTAR. Finally, the custom analysis of the Swift/BAT maps by Koss et al. (2013) shows a weak detection with flux $F_{14-195}=9.9_{-2.4}^{+5.1} \times 10^{-12} \mathrm{erg} \mathrm{s}^{-1} \mathrm{~cm}^{-2}$ consistent with $\mathrm{NuS}$ $T A R$. For instance, our model $\mathrm{T}$, when extrapolated to the energy range of 14-195 keV, implies best fit fluxes ranging over (8.49.3) $\times 10^{-12} \mathrm{erg} \mathrm{s}^{-1} \mathrm{~cm}^{-2}$ between the mission cross-calibration uncertainties.

Here, we examine the viability of the inferred long-term decline and its implications.

\subsubsection{On the possibility of contamination by another source}

Could the pre-BeppoSAX flux decline be associated with a contaminating source, unrelated to the AGN? The observed luminosities at the HEAO and Ginga epochs are above $10^{42} \mathrm{erg} \mathrm{s}^{-1}$ - already too high to be easily associated with X-ray binaries (XRBs) within the host galaxy. An XRB within our Galaxy which just happens to lie along the same 1.o.s. as NGC 7674 cannot be ruled out, though the high Galactic latitude $\left(b=-48^{\circ}\right)$ makes this unlikely. 


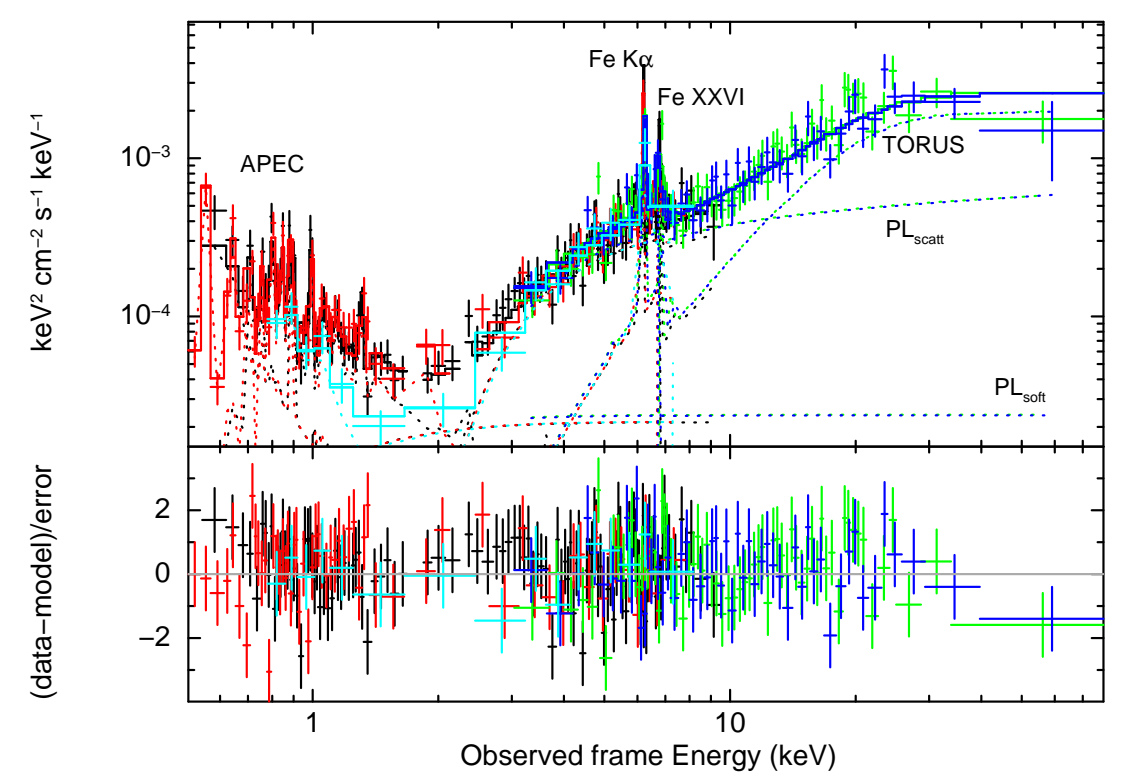

Figure 3. Suzaku, Swift and NuSTAR data fits with the reflection model T. Colours are as in Fig.2

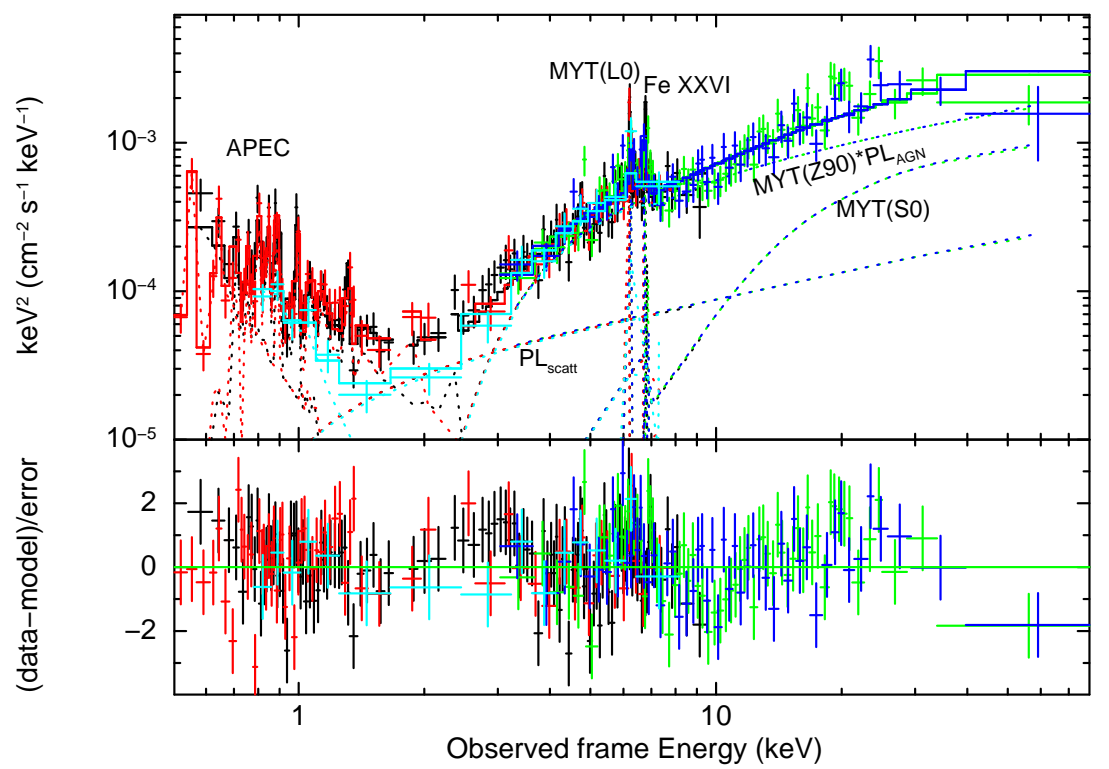

Figure 4. Decoupled MYTORUS model fit with the direct component absorber (MYTZ90) orientation being 90 deg, and the reflection being dominated by the face-on scatterer MYTS0 and its corresponding fluorescence emission from MYTL0.

Regarding contamination by other AGN, there is only one possible bright source in the recent 70 month all sky Swift/BAT hard $\mathrm{X}$-ray survey that could potentially have contaminated both the HEAO and Ginga fields of view. This is PKS 2325+093 which lies at a separation of $0.9 \mathrm{deg}$ from NGC 7674 and shows a BAT flux of $F_{14-195} \approx 3 \times 10^{-11} \mathrm{erg} \mathrm{s}^{-1} \mathrm{~cm}^{-2}$ (Baumgartner et al. 2013). However, this source lies outside the $H E A O$ positional error box of NGC 7674 (Bianchi et al. 2005). Moreover, PKS 2325+093 shows a very hard spectrum, at least in the BAT band, with $\Gamma_{\mathrm{BAT}}=1.29 \pm 0.28$. This is much harder than the spectral shape inferred for the Ginga observation below $\approx 10 \mathrm{keV}$ by Bianchi et al. (2005). Although a drastic change in spectral curvature around $10 \mathrm{keV}$ cannot be ruled out, the combined weight of evidence appears to disfavour contamination.

\subsubsection{Background uncertainties}

Measurements with non-imaging detectors such as the HEAO A1 Large Area Sky Survey instrument (Wood et al. 1984) and the Ginga Large Area Counter (Turner et al. 1989) are prone to uncertain background estimates, especially at low source flux levels. However, as discussed by Bianchi et al. (2005), the background for the observation of NGC 7674 is based on a scanning observation obtained close in time to the target (Awaki et al. 1991). These background scans result in noise estimates which are more reliable than model estimates typically adopted for non-imaging detectors.

Therefore, the Ginga flux, at least, is considered to be reliable and is significantly higher than that seen by subsequent missions, by about a factor of 10 . The HEAO flux, on the other hand, may 


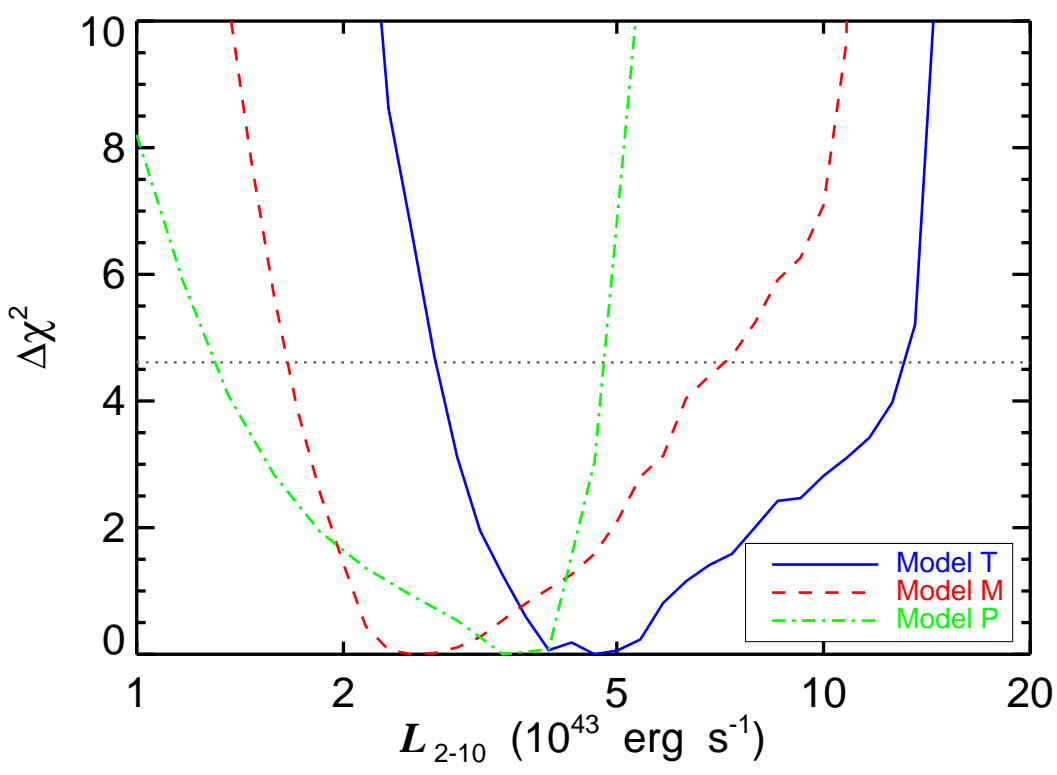

Figure 5. $\chi^{2}$ contours as a function of $L_{2-10}$ for models T, M and P. Luminosities are computed over the the two-dimensional parameter space of randomised photon index $\Gamma$ and normalisation $N$ values for $\mathrm{PL}_{\mathrm{AGN}}$, and the overall one-dimensional envelope of corresponding $\chi^{2}$ values is then plotted. The dotted horizontal line corresponds to $\Delta \chi^{2}=4.61$, or $90 \%$ confidence for the two starting parameters $\Gamma$ and $N$, relative to the minimum $\chi^{2}$ for each model.

well be affected by the above uncertainties, and we do not consider it as a strong constraint in the following discussion.

\subsubsection{The 'switched-off' AGN scenario}

Assuming that the luminosity change (at least that between Ginga and subsequent missions) is associated with the AGN in NGC 7674 itself, there are then two possible implications: the AGN could either have faded dramatically (an effective 'switch-off', as is referred to hereafter), or it could have become enshrouded within Compton-thick material after the Ginga observation. We consider these cases here.

The switched-off AGN scenario was examined by Bianchi et al. (2005), motivated by the fact that, unlike other changing-look AGN in which $N_{\mathrm{H}}$ variations occur relatively frequently, NGC 7674 did not show any flux or spectral variations in post-BeppoSAX observations. In this scenario, the observed reflection component dominating at hard X-rays is expected to be delayed with respect to the incident PL $_{\mathrm{AGN}}$ which has now faded. Similarly, the absorbed scattered component ( $\mathrm{PL}_{\text {scatt }}$ )' included in our models $\mathrm{T}$ and $\mathrm{M}$ (cf. Fig. 3) would also be interpreted as a delayed component scattered in to the 1.o.s from material on $\sim$ pc scales, or larger. The current PL $_{\mathrm{AGN}}$ flux level cannot be higher than that this $\mathrm{PL}_{\text {scatt }}$ component, which has a deabsorbed luminosity $L_{2-10}^{\text {scatt }} \approx 2 \times 10^{42} \mathrm{erg} \mathrm{s}^{-1}-$ an order of magnitude fainter than inferred during the Ginga observation epoch (1989). In model P, where no scattering component is required (cf. Fig.2), the constraint on the current intrinsic AGN luminosity is even more stringent, with $L_{2-10}$ expected to be 10 times lower still.

Assuming that the source switched off between the Ginga (1989) and BeppoSAX (1996) epochs around 1993, i.e. $21 \pm 3$ years before the NUSTAR observation, the absence of any flux change since then places a minimum limit on the radius $(R)$ of an axisymmetric reflector $R=3.2 \mathrm{pc}$ based upon a simple consideration of the light travel time from the far wall of a nearly edge-on reflector. Considering the average travel time over the full body of the reflector, and/or intermediate inclinations angles, would push up the lower limit on $R$. While extended reflectors on scales of up to $\sim 150$ pc have been observed in several CTAGN (e.g. NGC 4945 , Marinucci et al. 2012; Circinus, Arévalo et al. 2014; NGC 1068, Bauer et al. 2014), the studies so far find that these extended components make relatively minor flux contributions compared to the compact reflectors.

In fact, detailed studies of Type 1 AGN have shown that the bulk of the neutral $\mathrm{Fe} \mathrm{K} \alpha$ emission line arises at very compact scales of the dust sublimation radius $\left(R_{\text {sub }}\right)$ or smaller (see detailed discussion in Gandhi et al. 2015, and references therein), and in the orientation-based unification scheme, this would also hold for obscured and CTAGN viewed at higher inclination angles. For NGC 7674, $R_{\text {sub }}$ is estimated to be $\approx 0.1_{-0.04}^{+0.05} \mathrm{pc}$ based upon infrared luminosity scaling relations determined at high angular resolution where the AGN can be effectively isolated from surrounding star formation (Hönig et al. 2010). This size scale is about 30 times smaller than the lower limit on $R$ above.

In other words, in the switched-off AGN scenario, a typical (sub)-pc scale torus reflector would be expected to respond to a decline of the intrinsic continuum on timescales faster than seen in Fig.6 and we would have expected to see some change in the reflected component fluxes under the switched-off AGN scenario by now.

\subsubsection{Clumpy Compton-thick obscurer scenario}

In the obscured AGN scenario, the source became obscured sometime between the Ginga (1989) and the BeppoSAX (1996) epochs, and is now fully covered by CT material along the 1.o.s. Varying nuclear obscuration is common in AGN, but extreme transitions between Compton-thick and Compton-thin states are not 
(Markowitz et al. 2014). So it is interesting to examine whether the obscurer in NGC 7674 may somehow be atypical. Some qualitative constraints on the nature and geometry of the obscuring clouds are possible as follows.

Firstly, we argue that the global covering factor of the material is unlikely to be atypically low. This is because the reflection component we observe is strong. The observed reflected luminosity $\left(L_{\text {obs }}\right)$ scales approximately with intrinsic luminosity $\left(L_{\text {int }}\right)$ as

$L_{\mathrm{obs}} \sim L_{\mathrm{int}} \Omega a$

where $\Omega$ is the solid angle of the reflector and $a$ is the albedo. The physical models that we have used in our spectral fits assume fairly typical geometrical covering factors for the torus, and yield estimates of $L_{\text {int }}$ which are in reasonable agreement (to within a factor of a few) with other multiwavelength indicators $(\$ 4.1 .1$. If the reflector had small covering factor $\Omega$, the corresponding geometrical correction would imply much higher values of $L_{\text {int }}$.

The complete lack of recent X-ray flux variability also supports this. Whereas an extended distribution of clouds can naturally spread out and dampen variations in the reflected flux relative to any variability in the direct AGN radiation, this is not possible in the low covering factor limit. Indeed, most reflectiondominated CTAGN are observed to show little, or no flux variations at all (cf. two well-studied examples include Circinus Arévalo et al. 2014] and NGC 5643 [Annuar et al.2015], although sensitive observations have recently caught a transient column density change in NGC 1068 [Marinucci et al. 2016]). Sources where significant flux variability is observed (e.g. NGC 4945 [Puccetti et al. 2014], ESO 565-G019 [Gandhi et al. 2013], and IC 751 [Ricci et al. 2016]) tend to be mildly Compton-thick AGN or significantly clumpy, with the direct transmitted component being stronger than the reflection component over at least some portion of the hard X-ray regime. In particular, many observations support the presence of a small $\Omega$ for the obscurer in NGC 4945 (e.g. Madeiski et al. 2000, though there are other possible scenarios as discussed by Brightman et al. 2015).

The emerging scenario for NGC 7674 then is that a patchy distribution of clouds obscures the nucleus with a covering factor fairly typical of standard torus models. By the time of the BeppoSAX observation in 1996, a cloud ensemble with CT column density had fully obscured the 1.o.s to the nucleus, and this ensemble has continuously covered the 1.o.s. ever since.

Unlike changing-look AGN, however, NGC 7674 does not show frequent flux or spectral variability. This raises a complementary point of view that the past high state caught by Ginga was instead a transient near-complete unveiling of the nucleus caused by the 1.o.s passing through a hole in the patchy obscurer. Assuming relatively sharp optical depth edges to the obscuring clouds, the time gap of $\approx 7$ years between the Ginga and BeppoSAX observations is probably a strong upper limit to the time taken to cover the AGN X-ray emission region, which is expected to be highly compact (e.g. Risaliti et al.2007). In other words, the 'unveiling' could have been very brief, and Ginga may have simply been fortunate to catch the event.

Interestingly, converting the observed 1989 Ginga flux of $8( \pm 2) \times 10^{-12} \mathrm{erg} \mathrm{s}^{-1} \mathrm{~cm}^{-2}$ (Bianchi et al. 2005) to a luminosity yields $L_{2-10} \approx 2 \times 10^{43} \mathrm{erg} \mathrm{s}^{-1}$, again consistent with our spectral analysis results based upon the more recent data (Section 4.1). The effect of absorption in the Ginga spectrum appears minimal. This consistency provides additional support for the obscured AGN scenario.

Finally we note that, given the uncertainty associated with the $H E A O$ flux measurement, there is no strong constraint on the pre-Ginga flux evolution. If the $H E A O$ flux measurement were to be correct, the above analysis would imply that the AGN would have been intrinsically more luminous by a factor of $\approx 3$ during the $H E A O$ era. In this case, explaining the long-term flux evolution would require a combination of source fading (between $H E A O$ and Ginga) followed by patchy obscuration, which seems unlikely.

\subsection{On the weakness of the $\mathrm{Fe} \mathrm{K} \alpha$ line}

In our analysis, we found that the source has a relatively weak neutral $\mathrm{Fe} \mathrm{K} \alpha$ emission line, and showed that this can be reproduced by assuming either a low elemental abundance (model P), or an absorbed scattering solution (models $\mathrm{T}$ and $\mathrm{M}$ ). Here, we first place this result in context of other local CTAGN (Section 4.3.1) and then briefly investigate one other potential scenario of Iron line dilution by a jet (Section 4.3.2). In Section 4.3.3 we extend the comparison with other objects to higher redshifts (especially those that show prominent ionised $\mathrm{Fe}$ lines), before finally examining the broader implications of Fe line measurements in deep AGN surveys in Section 4.4

\subsubsection{Comparison with other bona fide CTAGN}

Compton-thick AGN are usually associated with strong $\mathrm{Fe} \mathrm{K} \alpha$ emission lines with equivalent widths $(\mathrm{EW})$ of $\gtrsim 1 \mathrm{keV}$. Our work shows a much weaker $\mathrm{EW} \approx 0.4 \mathrm{keV}$ (also reported by Bianchi et al. 2005), but our spectral modelling of NGC 7674 also consistently finds high Compton-thick column densities using several different spectral models.

In fact, comparing to other reflection-dominated AGN (such as NGC 1068, NGC 5643, Mrk 34 and others with $N_{\mathrm{H}} \geq 10^{25} \mathrm{~cm}^{-2}$ ) amongst the local bona fide CTAGN population (Della Ceca et al. 2008; Goulding et al. 2012; Gandhi et al. 2014), NGC 7674 appears to have one of the weakest neutral $\mathrm{Fe}$ fluorescence lines. This is demonstrated in Fig.77 where NGC 7674 stands out in the distribution of EW distribution of bona fide CTAGN compiled by Gandhi et al. (2014). The EW values are taken from relevant recent references in the same paper, or (where recent values are not published) from the compilation of Della Ceca et al. (2008). They are mostly based upon power law fits to the continuum together with Gaussian line components. Three sources have been excluded because of published works questioning their bona fide CTAGN nature, or showing that they have highly complex geometries: Mrk 3 $(\mathrm{EW} \approx 1.0 \pm 0.3 \mathrm{keV}$; Yaqoob et al. 2015; Guainazzi et al. 2016), NGC 7582 (EW $\approx 0.6_{-0.1}^{+0.6} \mathrm{keV}$; Rivers et al. 2015); and NGC 4939 $\left(\mathrm{EW} \approx 0.5_{-0.2}^{+0.4} \mathrm{keV}\right.$; Maiolino et al. 1998). Their exclusion does not affect the relative position of NGC 7674 .

\subsubsection{Dilution by a jet?}

One other possibility may be that a jet is diluting the continuum underlying the $\mathrm{Fe} \mathrm{K} \alpha$ emission line and hence effectively weakening the observed EW. Such dilution has been inferred in several broadline radio galaxies (e.g. Eracleous et al. 2000), and also in broad absorption line quasars (e.g. Luo et al. 2013).

In the case of NGC 7674, however, we consider this possibility to be unlikely for several reasons. Although NGC 7674 is a known radio source, it is classified as radio quiet (Xu et al. 1999). Then there is the distinct lack of $\mathrm{X}$-ray variability at $2-10 \mathrm{keV}$ as probed 


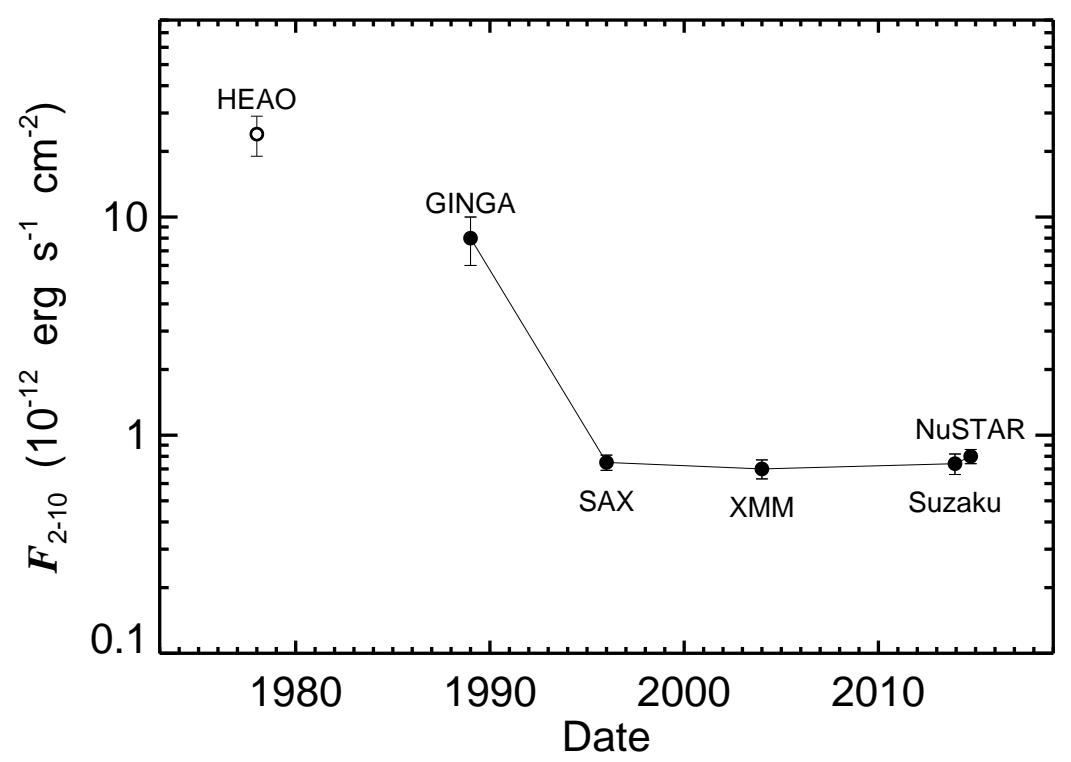

Figure 6. Long-term 2-10 keV observed flux light curve of NGC 7674 spanning 1977/78 (the HEAO epoch) to late 2014 (NUSTAR). The HEAO measurement is segregated from the other fluxes to stress its potentially uncertain nature.

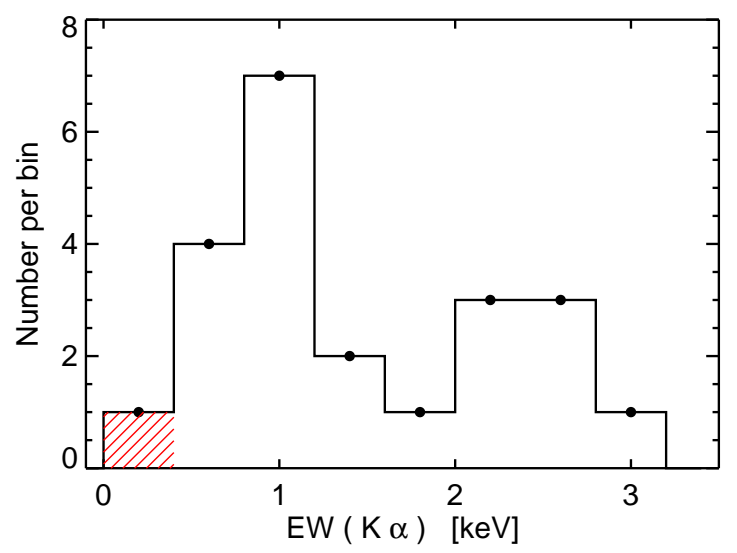

Figure 7. Distribution of equivalent widths (EWs) of the neutral $\mathrm{Fe} \mathrm{K} \alpha$ emission line for the bona fide CTAGN compiled in Gandhi et al. (2014). The red hatched region corresponds to NGC $7674\left(\mathrm{EW}=0.38_{-0.09}^{+0.10} \mathrm{keV}\right)$.

by several missions over the past $\sim 20$ years. Non-thermal emission from a jet is expected to be significantly variable, especially when probed on multiple (long) timescales that we are now able to sample (Fig.6).

Finally, one may try to quantify the expected flux from a jet irrespective of the above considerations. The core of NGC 7674 has a morphology extended over $\approx 1 \mathrm{kpc}$, characteristic of radio galaxies (e.g. Momjian et al. 2003). There is evidence of complex interactions of the radio ejecta with the interstellar medium - complex enough that the location of the AGN itself is unclear in the radio (Momiian et al. 2003). The reported integrated $5 \mathrm{GHz}$ nuclear radio flux density is $67 \mathrm{mJy}$ (Condon et al. 1991), corresponding to a monochromatic luminosity density of $L_{5 \mathrm{GHz}}=1.3 \times 10^{30} \mathrm{erg} \mathrm{s}^{-1} \mathrm{~Hz}^{-1}$.
The above luminosity is lower than the power typically associated with radio-loud (RLQs) as well as radio-intermediate quasars (RIQs). For example, Miller et al. (2011) studied samples of RIQs and RLQs and showed that there exists an 'X-ray excess' above that seen in radio-quiet sources. The excess scales with radio luminosity and could be associated with a jet contribution to X-rays. At the observed luminosity density of NGC 7674, the correlation between the X-ray excess and radio luminosity found by Miller et al. (2011) is consistent with no jet-related X-ray excess. Their correlation needs to be extended below their lower luminosity limit and would instead predict an X-ray deficit of $-0.14 \pm 0.09$ in their adopted units of $\ell_{x}-\ell_{x, \mathrm{RQQ}}$.

Instead of using the correlation for quasars, one may instead try using the correlation between radio power and the jet-related unabsorbed X-ray powerlaw luminosities for lower luminosity 3CRR sources presented by Hardcastle et al. (2009). In the terminology used by Hardcastle et al., the X-ray power law luminosity is $L_{\mathrm{X}, \mathrm{u}}$, with the $\mathrm{X}$-ray band being $\approx 0.4-8 \mathrm{keV}$, and a fixed spectral slope $\Gamma_{\text {jet }}=2$. From the reported $5 \mathrm{GHz}$ radio flux density, we predict $L_{\mathrm{X}, \mathrm{u}} \approx 10^{41} \mathrm{erg} \mathrm{s}^{-1}$, or $F_{2-10}=3 \times 10^{-14} \mathrm{erg} \mathrm{s}^{-1} \mathrm{~cm}^{-2}$. This is more than a factor of 20 fainter than our observed $F_{2-10}$. Accounting for a scatter of $0.56 \mathrm{dex}$ in the relation of Hardcastle et al. (2009), the predicted jet X-ray flux is still lower than the observed flux by a factor of 6 , rendering this scenario unlikely.

\subsubsection{On the strength of ionised Fe and the relation to other powerful infrared galaxies}

A variety of studies have found that distant sources with high bolometric power, including ultraluminous infrared galaxies (ULIRGs) and sub-mm galaxies, show preferentially strong ionised $\mathrm{Fe}$ lines such as FeXXV $(6.7 \mathrm{keV})$ and FeXXVI $(6.97 \mathrm{keV}$; e.g. Iwasawa et al. 2009; Lindner et al. 2012; Gilli et al. 2014). The neutral $\mathrm{Fe} \mathrm{K} \alpha(6.4 \mathrm{keV})$ line is extremely weak or undetected in many of these systems. This may be attributable either to the neu- 
tral reflection component being heavily embedded within the torus as seen by us, or to a highly ionised interstellar medium that is being energised by starburst activity, or a combination of the two effects. Iwasawa et al. (2012) further suggest that preferentially strong ionised emission lines may be connected to high accretion rates on the central AGN.

With a bolometric infrared power of $10^{11.56} \mathrm{~L}_{\odot}$ (Koss et al. 2013), NGC 7674 lies in the regime of luminous infrared galaxies. This is lower than, but approaching, the regime associated with ULIRG luminosities. Such an intermediate luminosity could explain why the source displays both a neutral and an ionised Fe line with strengths comparable to within a factor of about 2 . Our estimate of the Eddington ratio of the AGN in $\S 4.1$ is as high as $\approx 0.4$, and this could be even higher if $M_{\mathrm{BH}}$ has been overestimated (see Appendix). Brightman et al. (2016) also compile evidence showing that some CTAGN (with steep X-ray power law photon indices) may exhibit high Eddington fractions. If so, NGC 7674 would be a local analogue of the more bolometrically luminous systems with high accretion rates studied by Iwasawa et al. (2012). A more robust $M_{\mathrm{BH}}$ estimate will be needed to test these parallels.

Finally, we note that a strong ionised Fe XXVI emission feature may be accompanied by an ionised underlying continuum. So it is possible that the scattered component introduced in Section 3.2 actually arises from from photoionised or hot collisionally ionised gas which also self-consistently produces Fe XXVI and some of the soft emission. The possible need for a photoionised component was discussed in Section 4.1.2 We attempted to fit the data as such, by replacing the absorbed $\mathrm{PL}_{\text {scatt }}$ component from our base model $\mathrm{T}$ with a photoionised model based upon the publicly-available CLOUDY code (Ferland et al. 1998). Examples of such fits can be found for various other AGN, including Mrk 573 (Bianchi et al. 2010) and ESO 138-G001 (De Cicco et al. 2015). Reasonable fits were possible without $\mathrm{PL}_{\mathrm{scat}}$, but we found that the photoionised component was required to be extremely strong relative to the Compton-thick torus, and that it dominated over the entire energy range to up $\sim 10 \mathrm{keV}$. Although we cannot rule out such a model, photoionised components are usually much fainter (Bianchi et al. 2005; De Cicco et al. 2015). High spectral resolution observations will be required to test this scenario in further detail.

\subsection{Implications for identifying Compton-thick AGN at low $\mathrm{X}$-ray signal-to-noise}

The fact that NGC 7674 shows a weak Fe K $\alpha$ emission line, yet clearly prefers a Compton-thick l.o.s column, has potentially important implications for the study of more distant AGN in deep surveys where the signal-to-noise is typically much weaker than in our data. Using simulations, Koss et al. (2015) found that robust characterisation of typical nearby CTAGN such as NGC 3393 is only possible at relatively low redshifts of $z \lesssim 0.2$ if one relies upon detection of the $\mathrm{Fe} \mathrm{K} \alpha$ emission line and broadband continuum for spectral modelling.

Our results on NGC 7674 further complicate this issue. With a value of $\mathrm{EW}(\mathrm{Fe} \mathrm{K} \alpha)$ weaker by a factor of $\approx 3$ than in NGC 3393, it becomes even more difficult to identify an object as being Compton-thick. We demonstrate this by carrying out a simulation of a source with a model spectrum identical to NGC 7674, but with a lower flux of $F_{8-24}=5 \times 10^{-13} \mathrm{erg} \mathrm{s}^{-1} \mathrm{~cm}^{-2}$. Although four times fainter than NGC 7674 in the same band, this flux level lies more than an order of magnitude above the deepest flux level being probed in ongoing deep and wide NuSTAR surveys (Mullaney et al. 2015; Civano et al. 2015; Aird et al. 2015; Harrison et al. 2016).

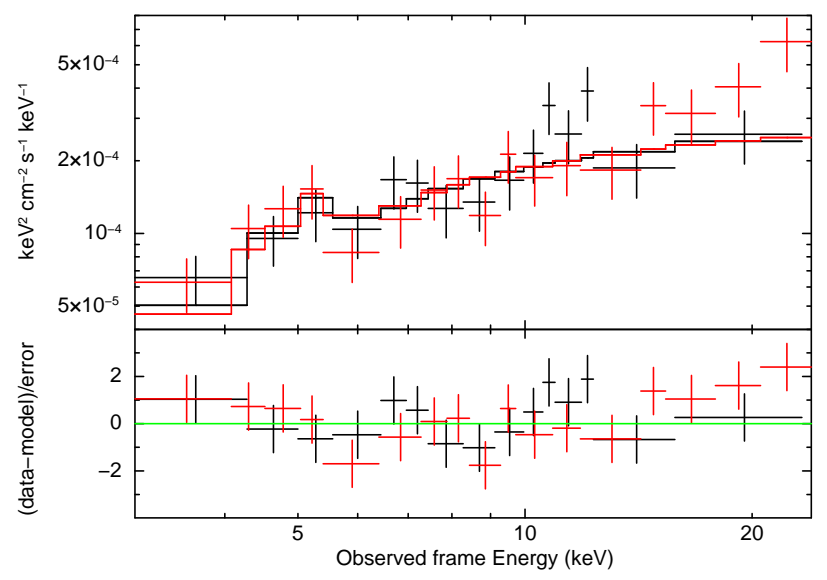

Figure 8. NUSTAR simulated spectra for two FPMs assuming our model T best fitting model to NGC 7674, but with a flux lower by a factor of 4 and shifted to $z=0.25$. The simulation is for $100 \mathrm{ks}$ of exposure time per FPM. The histograms show best fitting TORUS model to the faked data, and are fitted with $N_{\mathrm{H}}=2.2_{-0.8}^{+0.9} \times 10^{23} \mathrm{~cm}^{-2}$ and $\Gamma=1.8$ for canonical fixed values of $\theta_{\text {tor }}=60 \mathrm{deg}$ and $\theta_{\text {inc }}=87 \mathrm{deg}$, i.e. a Compton-thin solution. The fit statistic is $\chi^{2} /$ dof $=34 / 29$.

We simulated NUSTAR spectra for both FPMs using the default background and response simulation files ${ }^{32}$ provided by the $N u S$ TAR team, and assuming an exposure time of $100 \mathrm{ks}$. We also introduced a small redshift $z=0.25$, effectively assuming an intrinsic X-ray luminosity $L_{2-10} \approx 10^{45} \mathrm{erg} \mathrm{s}^{-1}$, characteristic of luminous AGN likely to be found in deep surveys.

The simulated spectra are shown in Fig. 8 The rising continuum slope (from the Compton hump of our baseline model T) is clearly visible. We first fitted the simulated data with a simple power law model (not shown), which yielded $\Gamma=1.1 \pm 0.2$ with $\chi^{2} / \mathrm{dof}=27 / 29$. No additional $\mathrm{Fe} \mathrm{K} \alpha$ emission line is required for the fit. This hard slope is suggestive of the need for obscuration. However, including redshifted photoelectric absorption and reflection with a TORUS model implies an $N_{\mathrm{H}}$ of only $2.2_{-0.8}^{+0.9} \times 10^{23} \mathrm{~cm}^{-2}$ for a fixed canonical $\Gamma=1.8$, i.e. a Comptonthin solution (the solution shown in the figure). This is a direct result of the lack of a strong $\mathrm{Fe} \mathrm{K} \alpha$ emission line.

The newer torus models are now being widely used by the community for self-consistent modelling of the nuclear obscuring material, and these have proven to be very successful at characterising objects well into the Compton-thick regime. However, most of the publicly available torus models do not allow varying elemental abundances which, as we discuss in $\S 3.2$ means that sources like NGC 7674 cannot be fit without additional complexity. In any case, such model complexity is often not viable for fitting low signalto-noise data, with the result that the column density, and hence intrinsic luminosity, of distant AGN may be underestimated.

A full assessment of the resultant bias in deep surveys is beyond the scope of the present paper, until the frequency of weak Fe K $\alpha$ CTAGN such as NGC 7674 can be established. Independent selection of large samples of CTAGN candidates based upon X-ray spectral diagnostics and on multiwavelength indicators (i.e. high ratios of $L_{\mathrm{MIR}}$ or $L_{[\mathrm{O} \mathrm{III}]}$ to observed $L_{2-10}$ ) could be an informative first step in this direction (cf. Rovilos et al. 2014). Similarly,

32 http://sc.nustar.caltech.edu 
we recommend the use of varying elemental abundances as an additional free parameter in model fitting when signal-to-noise allows.

If we are to make robust progress on the Compton-thick selection problem, multiple redundant cross-checking methods must be employed (the work of Brandt \& Alexander 2015 contains a recent, comprehensive analysis of various techniques in the literature).

\section{SUMMARY}

We have presented NUSTAR spectroscopy of the local reflectiondominated AGN NGC 7674. Together with unpublished Suzaku and Swift data, we carried out broadband X-ray modelling of the $0.5-78 \mathrm{keV}$ spectrum assuming three geometries of the nuclear obscurer/reflector. The best fitting model in all cases requires a nuclear column density of obscuring gas $N_{\mathrm{H}}$ (nuc) of at least $3 \times 10^{24} \mathrm{~cm}^{-2}$ and possibly much higher, with an absorption-corrected luminosity $L_{2-10}=(3-5) \times 10^{43} \mathrm{erg} \mathrm{s}^{-1}$ (Table 2), agreeing with mid-infrared continuum and forbidden optical $\left[\mathrm{O}_{\mathrm{III}}\right]$ emission line indicators. The full uncertainty range on $L_{2-10}$ spans $\approx(1-13) \times 10^{43} \mathrm{erg} \mathrm{s}^{-1}$ (Fig. 5). A relatively weak neutral $\mathrm{Fe} \mathrm{K} \alpha$ emission line $(\mathrm{EW} \approx 0.4 \mathrm{keV})$ at $6.4 \mathrm{keV}$ is seen, together with a comparatively strong ionised Fe line consistent with $6.97 \mathrm{keV}$ emitted by Fe XXVI. We explore a variety of scenarios to explain the line complex and suggest that NGC 7674 may be similar to more powerful ULIRGs which also show similar trends of line complexity, possibly related to a high accretion rate (Section 4.3.3.

We have presented an X-ray light curve spanning 37 years (Fig.6, and find that the observed source X-ray flux has remained constant for about 20 years, prior to which it was brighter by a factor of at least $\approx 10$ when observed by Ginga. A past HEAO detection was 3 times brighter still, but background uncertainties make this measurement less reliable. A faded/switched-off AGN scenario requires a reflector size of at least $3 \mathrm{pc}$, which is $\approx 30$ times larger than the dust sublimation radius of the canonical pc-scale torus in NGC 7674, and thus is not a preferred explanation for the observed fading (Section 4.2.3).

The alternative scenario is that a clumpy Compton-thick obscuring medium has been continuously obscuring the source for $\approx 21 \pm 3$ years since the mid 1990s. Unlike known changing-look AGN, however, NGC 7674 does not show frequent flux or spectral shape transitions, with none observed since the BeppoSAX observation. If a steady-state patchy obscurer does surround the nucleus, the past high state of NGC 7674 could have represented a temporary unveiling of the nucleus (Section 4.2.4). It is also noteworthy that the source has been an optical Seyfert 2 for more than 30 years, implying that the source has shown no evidence of being a 'changing-look' AGN over significant periods of time in the optical. The relation between the strong nuclear outflow and the $\mathrm{X}$-ray-obscuring medium, and whether the outflow is connected to the past fading, also remains to be investigated. Continued monitoring of the source will be important, as will high spatial and spectral resolution multiwavelength observations to understand these connections.

The weakness of the neutral $\mathrm{Fe} \mathrm{K} \alpha$ emission line implies that canonical torus covering factors and Solar metallicities cannot be used in order to derive absorption correction factors. This is relevant for surveys of distant, fainter AGN where the signal to noise of the data do not allow detailed fitting of individual sources and simplifying assumptions are introduced for spectral modelling (Section 4.4).

\section{ACKNOWLEDGEMENTS}

This research has made use of data from the NUSTAR mission, a project led by the California Institute of Technology, managed by the Jet Propulsion Laboratory, and funded by the National Aeronautics and Space Administration. We thank the NuSTAR Operations, Software, and Calibration teams for support with the execution and analysis of these observations. This research has made use of the NUSTAR Data Analysis Software (NuSTARDAS) jointly developed by the ASI Science Data Center (ASDC, Italy) and the California Institute of Technology (USA). This work made use of data supplied by the UK Swift Science Data Centre at the University of Leicester (Evans et al. 2009). P.G. thanks STFC for support (grant reference ST/J003697/2). A.C. and A.M. acknowledge support from the ASI/INAF grant I/037/12/0011/13. A.C. acknowledges the Caltech Kingsley visitor program. We acknowledge financial support from Majlis Amanah Rakyat (MARA) Malaysia (A.A.), the Science and Technology Facilities Council (STFC) grant ST/I0015731/1 (D.M.A) and ST/K501979/1 (G.B.L). W.N.B acknowledges California Institute of Technology (Caltech) $\mathrm{NuS}$ $T A R$ subcontract 44A-1092750. S.B. acknowledges financial contribution from the agreement ASI-INAF I/037/12/0. We also acknowledge NASA NuSTAR A01 Award NNX15AV27G (F.E.B.), CONICYT-Chile grants Basal-CATA PFB-06/2007 (F.E.B., C.R.), FONDECYT Regular 1141218 (F.E.B., C.R.), "EMBIGGEN" Anillo ACT1101 (F.E.B., C.R.), and the Ministry of Economy, Development, and Tourism's Millennium Science Initiative through grant IC120009, awarded to The Millennium Institute of Astrophysics, MAS (F.E.B.). We thank the referee for detailed comments which helped us to make the presentation of results clearer and to place the discussion on a more robust footing.

\section{A1 APPENDIX}

\section{A1.1 Individual Swift observations}

A total of 17 Swift XRT observations of NGC 7674 are available from the HEASARC archive 33 Their spectra were extracted from the standard data products, and fit with simple power laws. Fixed Galactic absorption was included in these fits. In cases of insignificant detection, the gross count rate at the source position was converted to an upper limit on the observed flux assuming a power law of fixed $\Gamma=1$ because we expect the source to be obscured and display an effectively hard photon index. The resultant fits are listed in Table A1 There is no evidence for significant variability amongst these measured fluxes.

\section{A1.2 Model M spectral fit}

Fig. A1 shows the fit to the default (coupled) model M incorporating MYTORUS $(\S 3.3)$. The fit parameters are listed in the last data column in Table 2 This fit is qualitatively very similar to that of model T, shown in Fig. 3

\section{A1.3 Black hole mass estimate from optical spectroscopy}

We use the penalised PiXel Fitting software (pPXF, Cappellari \& Emsellem 2004) to measure the central stellar velocity dispersion $(\sigma)$. For a stellar library, we used templates

33 http://heasarc.gsfc.nasa.gov/docs/archive.html 
Table A1. Swift XRT observations of NGC 7674.

\begin{tabular}{|c|c|c|c|c|c|}
\hline Date & $\begin{array}{l}\text { ObsID } \\
\text { (2) }\end{array}$ & $\begin{array}{c}\text { Exposure } \\
\text { ks } \\
(3)\end{array}$ & $\begin{array}{c}\text { Ct rate } \\
10^{-3} \mathrm{~s}^{-1} \\
\text { (4) }\end{array}$ & $\begin{array}{l}\Gamma \\
(5)\end{array}$ & $\begin{array}{r}\text { Flux } \\
10^{-12} \mathrm{erg} \mathrm{s}^{-1} \mathrm{~cm}^{-2} \\
(6)\end{array}$ \\
\hline $2011-01-28$ & 00040884001 & 1492 & $<20.0$ & $1^{f}$ & $<2.15$ \\
\hline 2011-10-03 & 00040884002 & 845 & $<13.8$ & $1^{f}$ & $<1.49$ \\
\hline 2011-10-04 & 00040884003 & 845 & $<26.7$ & $1^{f}$ & $<2.87$ \\
\hline $2011-10-28$ & 00040884004 & 4952 & $5.9 \pm 1.9$ & $-1.52_{-u}^{+1.55}$ & $2.13_{-1.09}^{+1.96}$ \\
\hline $2011-10-30$ & 00040884005 & 3696 & $7.9 \pm 2.7$ & $-0.23_{-1.16}^{+1.20}$ & $1.46_{-0.66}^{+1.11}$ \\
\hline $2011-11-03$ & 00040884006 & 2831 & $9.2 \pm 3.2$ & $-0.46_{-1.68}^{+1.16}$ & $1.63_{-0.92}^{+1.66}$ \\
\hline 2011-11-08 & 00040884007 & 2782 & $4.3 \pm 2.2$ & $-1.11 \pm u$ & $1.46_{-1.01}^{+3.01}$ \\
\hline 2011-11-11 & 00040884008 & 4701 & $5.1 \pm 1.9$ & $3.15_{-2.58}^{+3.00}$ & $0.94_{-0.36}^{+1.01}$ \\
\hline $2011-11-13$ & 00040884009 & 4997 & $5.8 \pm 1.9$ & $-0.03_{-1.32}^{+1.38}$ & $0.98_{-0.45}^{+0.85}$ \\
\hline $2011-11-15$ & 00040884010 & 3929 & $4.4 \pm 1.9$ & $-1.88_{-u}^{+2.21}$ & $2.40_{-1.60}^{+0.45}$ \\
\hline $2011-11-17$ & 00040884011 & 467 & $<25.4$ & $1^{-2}$ & $<2.73$ \\
\hline 2011-11-19 & 00040884012 & 5077 & $4.0 \pm 1.6$ & $-0.11_{-2.67}^{+2.70}$ & $0.99_{-0.48}^{+1.5}$ \\
\hline $2011-11-21$ & 00040884013 & 4679 & $3.8 \pm 1.6$ & $0.46_{-1.90}^{+1.84}$ & $0.94_{-0.44}^{+1.15}$ \\
\hline 2013-01-29 & 00049851001 & 1454 & $<9.5$ & $1^{-1}$ & $\begin{array}{l}-0.44 \\
<1.02\end{array}$ \\
\hline 2014-09-30 & 00080798001 & 1041 & $<21.7$ & $1^{f}$ & $<2.34$ \\
\hline $2014-10-03$ & 00080798002 & 3078 & $<9.1$ & $1^{f}$ & $<0.98$ \\
\hline $2014-10-08$ & 00080798003 & 2395 & $7.4 \pm 3.0$ & $0.55_{-1.86}^{+1.79}$ & $0.92_{-0.53}^{+1.59}$ \\
\hline
\end{tabular}

Ct rates (3), fitted $\Gamma$ (4) and flux (5) are for the $2-10 \mathrm{keV}$ range where the AGN is expected to dominate.

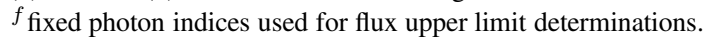
${ }^{u}$ unconstrained.

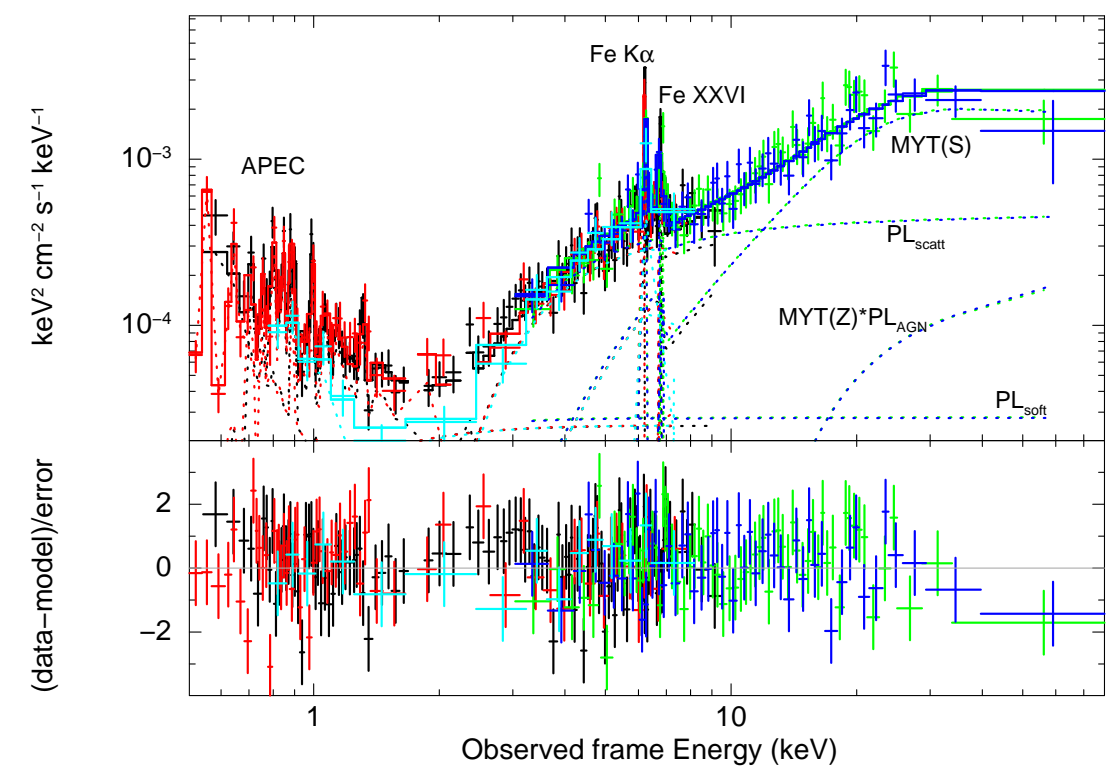

Figure A1. Suzaku + NuSTAR data fits with the reflection model M. This is very similar to model T presented in Fig. 3 The MYT(Z) and MYT(S) components represent the absorption of the direct component, and the Compton-scattered component, respectively. The third MYTORUS component is the fluorescence component producing the $\mathrm{Fe} \mathrm{K} \alpha$ and related emission features and the Compton shoulders (this would be equivalently named MYTL).

from the Miles Indo-U.S. Catalogue (MIUSCAT) library of stellar spectra (Vazdekis et al. 2012) with coverage of the wavelength range of the Ca II triplet (8450-8700 $)$. The data and fit are presented in Fig. $\mathrm{A} 2$ The fit yielded a stellar velocity dispersion of $\sigma=91 \pm 48 \mathrm{~km} \mathrm{~s}^{-1}$ (1-sigma uncertainty). This value is entirely consistent with, and slightly below, the instrumental resolution $\sigma_{\text {instr. }}=107 \mathrm{~km} \mathrm{~s}^{-1}$. Measurements near the resolution limit can be unreliable, so we conservatively interpret the measurement and its uncertainty to be equivalent to an upper limit of $\sigma=91+48=139 \mathrm{~km} \mathrm{~s}^{-1}$. Using the $M_{\mathrm{BH}}-\sigma$ relation from McConnell \& Ma (2013) implies an upper limit of $M_{\mathrm{BH}}<10^{7.43} \mathrm{M}_{\odot}$.

The measurement quoted by Nelson \& Whittle (1995) is $\sigma=144 \pm 32 \mathrm{~km} \mathrm{~s}^{-1}$, which has been used to infer $M_{\mathrm{BH}}=10^{7.56} \mathrm{M}_{\odot}$ in the literature (Bian \& Gu 2007). Nelson \& Whittle used the KPNO $2.1 \mathrm{~m}$ telescope with the TI CCD, a slit width of $1^{\prime \prime} .5$, and 600 lines $\mathrm{mm}^{-1}$ grating, observed under $1^{\prime \prime} 9$ seeing. The grating would have had higher spectral resolution than our LRIS observations, so it is a bit surprising that their measurement of $\sigma$ is larger than our inferred upper limit. 


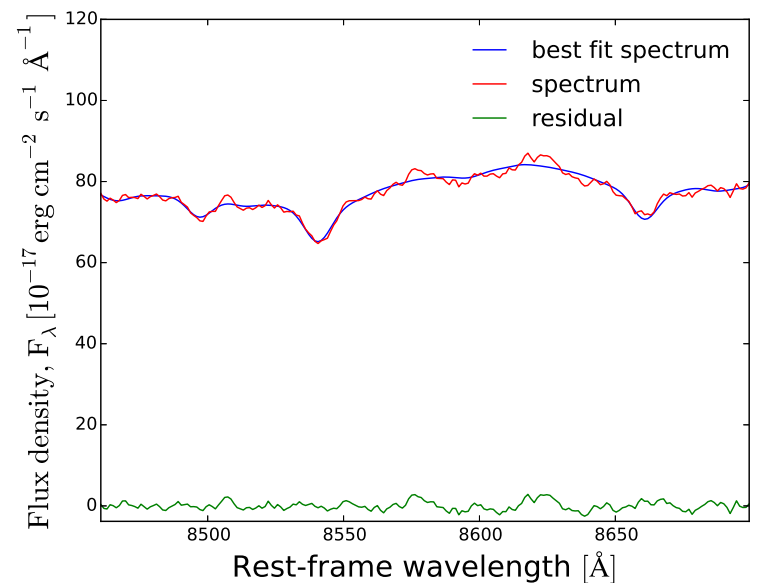

Figure A2. Keck/LRIS red grism spectrum of NGC 7674 spanning the Ca II triplet absorption feature, fitted with a pPXF algorithm. The intrinsic velocity dispersion is measured to be $91 \pm 48 \mathrm{~km} \mathrm{~s}^{-1}$.

However, we note that the uncertainty of $32 \mathrm{~km} \mathrm{~s}^{-1}$ suggests that the discrepancy is relatively mild. Their use of a wider slit, the bad seeing during their observation, and use of a far smaller telescope than Keck may have all resulted in lower signal-to-noise than our data. In fact, a comparison by eye of our Fig. A2 with the spectrum presented in Fig. 3a of Nelson \& Whittle (1995) shows this to be a plausible solution to the above mismatch. A sensitive, higher resolution optical spectrum, with 1200 lines $\mathrm{mm}^{-1}$, for instance, should be able to resolve this issue.

\section{REFERENCES}

Aird J. et al., 2015, ApJ, 815, 66

Annuar A. et al., 2015, ApJ, 815, 36

Arévalo P. et al., 2014, ApJ, 791, 81

Arnaud K. A., 1996, in ASP Conf. Ser. 101: Astronomical Data Analysis Software and Systems V, eds. George H. Jacoby and Jeannette Barnes, Vol. 5, p. 17

Asmus D., Gandhi P., Hönig S. F., Smette A., Duschl W. J., 2015, MNRAS, 454, 766

Asmus D., Hönig S. F., Gandhi P., Smette A., Duschl W. J., 2014, MNRAS, 439, 1648

Awaki H., Koyama K., Inoue H., Halpern J. P., 1991, PASJ, 43, 195

Ballantyne D. R., Draper A. R., Madsen K. K., Rigby J. R., Treister E., 2011, ApJ, 736, 56

Baloković M. et al., 2014, ApJ, 794, 111

Barthelmy S. D. et al., 2005, Sp. Sc. Rev., 120, 143

Bassani L., Dadina M., Maiolino R., Salvati M., Risaliti G., della

Ceca R., Matt G., Zamorani G., 1999, ApJS, 121, 473

Bauer F. E. et al., 2014, ApJ submitted, arXiv:1411.0670

Baumgartner W. H., Tueller J., Markwardt C. B., Skinner G. K., Barthelmy S., Mushotzky R. F., Evans P. A., Gehrels N., 2013, ApJS, 207, 19

Bian W., Gu Q., 2007, ApJ, 657, 159

Bianchi S., Chiaberge M., Evans D. A., Guainazzi M., Baldi R. D., Matt G., Piconcelli E., 2010, MNRAS, 405, 553

Bianchi S., Guainazzi M., Matt G., Chiaberge M., Iwasawa K., Fiore F., Maiolino R., 2005, A\&A, 442, 185
Blackburn J. K., 1995, in Astronomical Society of the Pacific Conference Series, Vol. 77, R. A. Shaw, H. E. Payne, \& J. J. E. Hayes , ed, Astronomical Data Analysis Software and Systems IV, p. 367

Bohlin R. C., Savage B. D., Drake J. F., 1978, ApJ, 224, 132

Brandt W. N., Alexander D. M., 2015, A\&AR, 23, 1

Brightman M. et al., 2015, ApJ, 805, 41

Brightman M. et al., 2016, ApJ, 826, 93

Brightman M., Nandra K., 2011, MNRAS, 413, 1206

Burrows D. N. et al., 2005, Sp. Sc. Rev., 120, 165

Cappellari M., Emsellem E., 2004, PASP, 116, 138

Cappi M. et al., 2006, A\&A, 446, 459

Civano F. et al., 2015, ApJ, 808, 185

Comastri A., Setti G., Zamorani G., Hasinger G., 1995, A\&A, 296, 1

Condon J. J., Frayer D. T., Broderick J. J., 1991, AJ, 101, 362

Cutri R. M. et al., 2013, Explanatory Supplement to the AllWISE

Data Release Products, Technical report

De Cicco M., Marinucci A., Bianchi S., Piconcelli E., Violino G., Vignali C., Nicastro F., 2015, MNRAS, 453, 2155

Della Ceca R. et al., 2008, Mem SAI, 79, 65

Dickey J. M., Lockman F. J., 1990, ARA\&A, 28, 215

Eracleous M., Sambruna R., Mushotzky R. F., 2000, Hard X-Ray Spectra of Broad-Line Radio Galaxies from the Rossi X-Ray Timing Explorer

Evans P. A. et al., 2009, MNRAS, 397, 1177

Feldman F. R., Weedman D. W., Balzano V. A., Ramsey L. W., 1982, ApJ, 256, 427

Ferland G. J., Korista K. T., Verner D. A., Ferguson J. W., Kingdon J. B., Verner E. M., 1998, PASP, 110, 761

Fischer T. C., Crenshaw D. M., Kraemer S. B., Schmitt H. R., 2013, ApJS, 209, 1

Fukazawa Y. et al., 2009, PASJ, 61, 17

Gandhi P., Fabian A. C., 2003, MNRAS, 339, 1095

Gandhi P., Hönig S. F., Kishimoto M., 2015, ApJ, 812, 113

Gandhi P., Horst H., Smette A., Hönig S., Comastri A., Gilli R., Vignali C., Duschl W., 2009, A\&A, 502, 457

Gandhi P. et al., 2014, ApJ, 792, 117

Gandhi P. et al., 2013, ApJ, 773, 51

Gehrels N. et al., 2004, ApJ, 611, 1005

Gilli R., Comastri A., Hasinger G., 2007, A\&A, 463, 79

Gilli R. et al., 2014, A\&A, 562, A67

Goulding A. D., Alexander D. M., Bauer F. E., Forman W. R., Hickox R. C., Jones C., Mullaney J. R., Trichas M., 2012, ApJ, 755,5

Grossan B. A., 1992, Ph.D. thesis, Massachusetts Institute of Technology, Cambridge.

Guainazzi M., Bianchi S., 2007, MNRAS, 374, 1290

Guainazzi M. et al., 2016, MNRAS in press, arXiv:1605.02467

Hardcastle M. J., Evans D. A., Croston J. H., 2009, MNRAS, 396, 1929

Harrison F. A. et al., 2016, ApJ, 831, 185

Harrison F. A. et al., 2013, ApJ, 770, 103

Hönig S. F., Kishimoto M., Gandhi P., Smette A., Asmus D.,

Duschl W., Polletta M., Weigelt G., 2010, A\&A, 515, A23

Iwasawa K. et al., 2012, A\&A, 537, A86

Iwasawa K., Sanders D. B., Evans A. S., Mazzarella J. M., Armus

L., Surace J. A., 2009, ApJL, 695, L103

Kennicutt R. C., Jr., 1998, ARA\&A, 36, 189

Kinkhabwala A. et al., 2002, ApJ, 575, 732

Kokubun M. et al., 2007, PASJ, 59, 53 
Konami S., Matsushita K., Gandhi P., Tamagawa T., 2012, PASJ, 64, 117

Koss M., Mushotzky R., Baumgartner W., Veilleux S., Tueller J., Markwardt C., Casey C. M., 2013, ApJL, 765, L26

Koss M. J. et al., 2016, ApJ in press, arXiv:1604.07825

Koss M. J. et al., 2015, ApJ, 807, 149

Koyama K. et al., 2007, PASJ, 59, 23

Lamastra A., Bianchi S., Matt G., Perola G. C., Barcons X., Carrera F. J., 2009, A\&A, 504, 73

Lansbury G. B. et al., 2015, ApJ, 809, 115

Lindner R. R., Baker A. J., Beelen A., Owen F. N., Polletta M., 2012, ApJ, 757, 3

Liu Y., Li X., 2015, MNRAS, 448, L53

Luo B. et al., 2013, ApJ, 772, 153

Madejski G., Życki P., Done C., Valinia A., Blanco P., Rothschild

R., Turek B., 2000, ApJL, 535, L87

Magdziarz P., Zdziarski A. A., 1995, MNRAS, 273, 837

Maiolino R., Salvati M., Bassani L., Dadina M., della Ceca R.,

Matt G., Risaliti G., Zamorani G., 1998, A\&A, 338, 781

Malaguti G. et al., 1998, A\&A, 331, 519

Marinucci A. et al., 2016, MNRAS, 456, L94

Marinucci A., Risaliti G., Wang J., Nardini E., Elvis M., Fabbiano

G., Bianchi S., Matt G., 2012, MNRAS, 423, L6

Markowitz A. G., Krumpe M., Nikutta R., 2014, MNRAS, 439, 1403

Mateos S. et al., 2005, A\&A, 433, 855

Matt G., Guainazzi M., Maiolino R., 2003, MNRAS, 342, 422

McConnell N. J., Ma C.-P., 2013, ApJ, 764, 184

Miller B. P., Brandt W. N., Schneider D. P., Gibson R. R., Steffen A. T., Wu J., 2011, ApJ, 726, 20

Mineo S., Gilfanov M., Sunyaev R., 2012, MNRAS, 426, 1870

Momjian E., Romney J. D., Carilli C. L., Troland T. H., 2003, ApJ, 597, 809

Mullaney J. R. et al., 2015, ApJ, 808, 184

Murphy K. D., Yaqoob T., 2009, MNRAS, 397, 1549

Nandra K., George I. M., Mushotzky R. F., Turner T. J., Yaqoob T., 1997, ApJL, 488, L91

Nandra K., O’Neill P. M., George I. M., Reeves J. N., 2007, MNRAS, 382, 194

Nelson C. H., Whittle M., 1995, ApJS, 99, 67

Oke J. B. et al., 1995, PASP, 107, 375

Piconcelli E., Jimenez-Bailón E., Guainazzi M., Schartel N., Rodríguez-Pascual P. M., Santos-Lleó M., 2005, A\&A, 432, 15

Planck Collaboration , 2014, A\&A, 571, A16

Puccetti S. et al., 2014, ApJ, 793, 26

Ranalli P., Comastri A., Origlia L., Maiolino R., 2008, MNRAS, 386, 1464

Ranalli P., Comastri A., Setti G., 2003, A\&A, 399, 39

Ricci C. et al., 2016, ApJ, 820, 5

Ricci C., Ueda Y., Koss M. J., Trakhtenbrot B., Bauer F. E., Gandhi P., 2015, ApJL, 815, L13

Risaliti G., Elvis M., Fabbiano G., Baldi A., Zezas A., 2005, ApJL, 623, L93

Risaliti G., Elvis M., Fabbiano G., Baldi A., Zezas A., Salvati M., 2007, ApJL, 659, L111

Rivers E. et al., 2015, ApJ, 815, 55

Rovilos E. et al., 2014, MNRAS, 438, 494

Sako M., Kahn S. M., Paerels F., Liedahl D. A., 2000, ApJL, 543, L115

Setti G., Woltjer L., 1989, A\&A, 224, L21

Smith R. K., Brickhouse N. S., Liedahl D. A., Raymond J. C., 2001, ApJL, 556, L91
Stern D., 2015, ApJ, 807, 129

Stern D. et al., 2012, ApJ, 753, 30

Takahashi T. et al., 2007, PASJ, 59, 35

Telesco C. M., Harper D. A., 1980, ApJ, 235, 392

Treister E., Urry C. M., Virani S., 2009, ApJ, 696, 110

Turner M. J. L. et al., 1989, PASJ, 41, 345

Ueda Y., Akiyama M., Hasinger G., Miyaji T., Watson M. G., 2014, ApJ, 786, 104

Unger S. W. et al., 1988, MNRAS, 234, 745

Vasudevan R. V., Fabian A. C., 2007, MNRAS, 381, 1235

Vazdekis A., Ricciardelli E., Cenarro A. J., Rivero-González J. G., Díaz-García L. A., Falcón-Barroso J., 2012, MNRAS, 424, 157

Wood K. S. et al., 1984, ApJS, 56, 507

Wright E. L. et al., 2010, AJ, 140, 1868

Xu C., Livio M., Baum S., 1999, AJ, 118, 1169

Yaqoob T., 2012, MNRAS, 423, 3360

Yaqoob T., Tatum M. M., Scholtes A., Gottlieb A., Turner T. J., 2015, MNRAS, 454, 973

\section{AUTHOR AFFILIATIONS}

${ }^{1}$ Department of Physics \& Astronomy, University of Southampton, Highfield, Southampton SO17 1BJ

${ }^{2}$ Centre for Extragalactic Astronomy, Department of Physics, Durham University, South Road, Durham DH1 3LE

${ }^{3}$ Institute of Astronomy, University of Cambridge, Madingley Road, Cambridge CB3 OHA

${ }^{4}$ Jet Propulsion Laboratory, California Institute of Technology, 4800 Oak Grove Drive, Mail Stop 169-221, Pasadena, CA 91109, USA

${ }^{5}$ Instituto de Astrofísica and Centro de Astroingeniería, Facultad de Física, Pontificia Universidad Católica de Chile, Casilla 306, Santiago 22, Chile

${ }^{6}$ Millennium Institute of Astrophysics (MAS), Nuncio Monseñor Sótero Sanz 100, Providencia, Santiago, Chile

${ }^{7}$ Space Science Institute, 4750 Walnut Street, Suite 205, Boulder, Colorado 80301, USA

${ }^{8}$ Dipartimento di Matematica e Fisica, Università degli Studi Roma Tre, via della Vasca Navale 84, 00146 Roma, Italy

${ }^{9}$ Space Sciences Laboratory, University of California, Berkeley, CA 94720, USA

${ }^{10}$ Department of Astronomy and Astrophysics, 525 Davey Lab, The Pennsylvania State University, University Park, PA 16802, USA

${ }^{11}$ Institute for Gravitation and the Cosmos, The Pennsylvania State University, University Park, PA 16802, USA

${ }^{12}$ Department of Physics, Pennsylvania State University, University Park, PA 16802, USA

${ }^{13}$ Cahill Center for Astrophysics, 1216 East California, Boulevard, California Institute of Technology, Pasadena, CA 91125, USA

${ }^{14}$ DTU Space-National Space Institute, Technical University of Denmark, Elektrovej 327, DK-2800 Lyngby, Denmark

${ }^{15}$ INAF Osservatorio Astronomico di Bologna via Ranzani 1, 40127 Bologna Italy

${ }^{16}$ Lawrence Livermore National Laboratory, Livermore, CA 94550, USA

${ }^{17}$ Max-Planck-Institut für Extraterrestrische Physik (MPE), Postfach 1312, D-85741 Garching, Germany

${ }^{18}$ Harvard-Smithsonian Center for Astrophysics, 60 Garden Street, Cambridge, MA 02138, USA

${ }^{19}$ Institute of Space and Astronatical Science (JAXA), 3-1-1 Yoshinodai, Sagamihara, Kanagawa, 252-5252, Japan

${ }^{20}$ European Space Astronomy Center of ESA, P.O.Box 78, Villanueva de la Cañada, E-28691 Madrid, Spain 
${ }^{21}$ Columbia Astrophysics Laboratory, $550 \mathrm{~W}$ 120th Street, Columbia University, NY 10027, USA

${ }^{22}$ Institute for Astronomy, Department of Physics, ETH Zurich, Wolfgang-Pauli-Strasse 27, CH-8093 Zurich, Switzerland

${ }^{23}$ INAF Istituto di Astrofisica Spaziale e Fisica cosmica di Bologna, via Gobetti 101, I-40129, Bologna, Italy

${ }^{24}$ Dipartimento di Fisica e Astronomia (DIFA), Università di Bologna, viale Berti Pichat 6/2, 40127 Bologna, Italy

${ }^{25}$ ASDC-ASI, Via del Politecnico, 00133 Roma, Italy

${ }^{26}$ INAF-Osservatorio Astronomico di Roma, via Frascati 33, 00040 Monte Porzio Catone (RM), Italy

${ }^{27} X$-ray Astrophysics Laboratory, NASA Goddard Space Flight

Center, Greenbelt, MD 20771, USA 